Sensing

Elsevier Editorial System(tm) for ISPRS Journal of Photogrammetry and Remote Manuscript Draft

Manuscript Number: PHOTO-D-15-00150R2

Title: An object-based approach to delineate wetlands across landscapes of varied disturbance with high spatial resolution satellite imagery

Article Type: Original Research Paper

Keywords: GEOBIA, wetlands, landscape heterogeneity, multi-scale segmentation, high spatial resolution imagery

Corresponding Author: Dr. Amy B Mui, PhD

Corresponding Author's Institution: University of Toronto

First Author: Amy B Mui, PhD

Order of Authors: Amy B Mui, PhD; Yuhong He, PhD; Qihao Weng, PhD 
4

\title{
An object-based approach to delineate wetlands across landscapes of varied disturbance with high spatial resolution satellite imagery
}

\author{
Amy Mui ${ }^{\mathrm{a}}$, Yuhong $\mathrm{He}^{\mathrm{a}}$, Qihao Weng ${ }^{\mathrm{b}}$ \\ ${ }^{a}$ Department of Geography, University of Toronto Mississauga, 3359 Mississauga Road, \\ Mississauga, Ontario, L5L 1C6, Canada \\ ${ }^{\mathrm{b}}$ Center for Urban and Environmental Change, Department of Earth \& Environmental \\ Systems, Indiana State University, Terre Haute, IN 47809, USA
}

\section{Acknowledgements:}

This work was supported by an Industrial Postgraduate Scholarship from the National Science and Engineering Research Council (NSERC) to A.Mui sponsored by the Toronto Zoo, an NSERC Discovery Grant to Y.He, Graduate Expansion Funding from the Department of Geography at the University of Toronto Mississauga, and the Centre for Global Change Science (CGCS) at the University of Toronto. Additional funding was provided by the Environment Canada Habitat Stewardship Fund, the Province of Ontario, Ministry of Natural Resources, and the Town of Huntsville. The authors gratefully acknowledge B. Steinberg from the Ministry of Natural Resources, V. Leck from Parks Canada, and F. Dorombozi from Brant County for providing data and field support. 


\section{Research Highlights:}

- GEOBIA and high spatial resolution imagery can detect wetlands with an accuracy over $80 \%$.

- The multi-scale GEOBIA approach is particularly effective for wetland detection.

- Addition of texture, elevation, and NDVI data improves segmentation.

- A higher landscape heterogeneity may negatively affect wetland classification.

\section{Abstract}

Mapping wetlands across both natural and human-altered landscapes is important for the management of these ecosystems. Though they are considered important landscape elements providing both ecological and socioeconomic benefits, accurate wetland inventories do not exist in many areas. In this study, a multi-scale geographic object-based image analysis (GEOBIA) approach was employed to segment three high spatial resolution images acquired over landscapes of varying heterogeneity due to human-disturbance to determine the robustness of this method to changing scene variability. Multispectral layers, a digital elevation layer, normalised-difference vegetation index (NDVI) layer, and a first-order texture layer were used to segment images across three segmentation scales with a focus on accurate delineation of wetland boundaries and wetland components. Each ancillary input layer contributed to improving segmentation at different scales. Wetlands were classified using a nearest neighbour approach across a relatively undisturbed park site and an agricultural site using GeoEye1 imagery, and an urban site using WorldView2 data. Successful wetland classification was achieved across all study sites with an accuracy above $80 \%$, though results suggest that overall a higher degree of landscape heterogeneity may negatively affect both segmentation and classification. The agricultural site suffered from the greatest amount of over and under segmentation, and lowest map accuracy (kappa: 0.78) which was partially attributed to confusion among a greater proportion of mixed vegetated classes from both wetlands and uplands. Accuracy of individual wetland classes based on the Canadian Wetland Classification system varied between each site, with kappa values ranging from 0.64 for the swamp class and 0.89 for the marsh class. This research developed a unique approach to mapping wetlands of various degrees of disturbance using GEOBIA, which can be applied to study other wetlands of similar settings. 
63 Keywords: GEOBIA, wetlands, landscape heterogeneity, multi-scale segmentation, high spatial 64 resolution imagery

\section{Introduction}

66 Mapping wetlands across natural and human-altered landscapes is important for understanding

67 their responses to natural and anthropogenic activities, for developing strategies to conserve

68 wetland biodiversity, and to prioritise areas for restoration or protection. While public perception

69 of the conservation value of wetlands has increased over the past century (Brock et al., 1999),

70 wetland loss appears to continue with little abatement and this change requires ongoing

71 monitoring.

72

73 The ability to delineate wetlands and monitor changes in a semi-automated, and ongoing manner is

74 important to the management of these ecosystems. A viable approach is the use of satellite remote

75 sensing data, which provides advantages of large area coverage, ongoing data collection, and

76 improved spatial resolution for wetland detection. While a variety of methods to delineate wetlands

77 have been used with varying success (Davranche et al., 2010; Hirano et al., 2003; Schmidt \&

78 Skidmore, 2003; Shanmugam et al., 2006), less attention has been given to the applicability of such

79 methods across different landscapes. Urban and rural landscapes represent uplands subjected to

80 disturbance related to increased surface heterogeneity, changes to hydrologic regime, and land

81 cover composition which may affect wetland detection accuracy.

82

83 Previous research has demonstrated that wetlands can be detected within upland surroundings, yet

84 a unified approach to mapping wetlands across landscapes of varying complexity has not been

85 identified. Further, fewer studies have included the detection of small and ephemeral wetlands

86 even though pools as small as 0.2 ha represent important, often critical habitat (Semlitsch \& Bodie,

87 1998). In some areas such as the glaciated prairie pothole region of central Canada, almost $88 \%$ of

88 wetlands are less than 0.4 ha in area (Halabisky, 2011). Coarser $30 \mathrm{~m}$ data such as those from the

89 Landsat constellation require a minimum of 9 pure pixels ( 0.9 ha) to consistently identify a feature

90 (Ozesmi \& Bauer, 2002), resulting in many mixed pixels and small wetlands below this threshold

91 being missed (Klemas, 2011; Powers et al. 2011). At the local scale, protection of small wetlands is

92 vital, particularly for the maintenance of biodiversity (Gibbs, 1993; Semlitsch \& Bodie, 1998), and

93 many wetlands in altered landscapes are significantly reduced in size from their former coverage. 
While the current cost associated with obtaining high spatial resolution satellite data can be high, the cost is still significantly lower than field surveying or aerial photographs (see Wei \& ChowFraser, 2007 for a cost breakdown) and provides the advantage of repeat coverage for monitoring 97 over time and the addition of data outside of the optical range (e.g., in the near infrared region). 98 Current work with high spatial resolution sensors has been used to successfully monitor the change in aquatic vegetation in coastal marshes (Wei \& Chow-Fraser, 2007), to discriminate between submerged and emergent wetland vegetation (Davranche et al., 2010), and to estimate marshland composition and biomass in riparian marshes (Dillabaugh \& King, 2008).

High resolution data provides the needed spatial resolution to capture smaller wetlands, but it also results in greater within-class spectral variance, making separation of mixed and similar land cover classes more difficult than with coarser-resolution imagery (Klemas, 2011; Hu \& Weng, 2011). To address this increased variance an appropriate classification method must be employed. In recent decades object based image analysis (OBIA), or geographic object based image analysis (GEOBIA), has gained much attention as an alternative to traditional pixel-based methods. The packaging of pixels into discrete objects minimizes the variance (noise) experienced by high spatial resolution images, allowing the objects, rather than individual pixels to be classified. Past work has found that the object-based approach is preferred over the pixel-based approach for 112 classifying urban areas (Myint et al., 2011; Hu \& Weng, 2011), mapping land cover (Whiteside \& 113 Ahmad, 2005; Yan et al., 2006), and land cover change (Dingle-Robertson \& King, 2011). The object-based approach has also been successfully used in wetland research for classifying macrophyte communities in coastal marsh habitat (Midwood \& Chow-Fraser, 2010; Rokitnicki116 Wojcik et al, 2011), evaluating the structure of patterned peatlands (Dissanska, Bernier, \& Payette, 117 2009), and mapping multiple classes of wetlands according to the Canadian Wetland Inventory 118 (Grenier et al., 2007). Fournier et al. (2007) reviewed wetland mapping methods to be applied to 119 the Canadian Wetlands Inventory program and identified the object-based approach as most 120 appropriate due to its flexibility and ability to address the spatial heterogeneity of wetlands. 121 Despite past successes in mapping wetland classes and vegetative communities, the majority of 122 previous research has focussed on wetlands by masking out the surrounding upland matrix in order 123 to concentrate on methods of within wetland classification. Yet the ability to delineate wetlands 124 from regions where a previous wetland inventory does not exist, is important for monitoring trends 125 and mitigating further wetland losses. 
Approaches to classification have ranged from traditional unsupervised (Sawaya et al., 2003; Jensen et al., 1995) and supervised algorithms (Wang et al., 2004; Yu et al., 2006) including fuzzy methods (Benz et al., 2004; Townsend \& Walsh, 2001) and object-based approaches (Blaschke, 2010; Blaschke et al. 2014) to more complex machine learning algorithms such as classification tree methods (Midwood \& Chow-Fraser, 2010; Wright \& Gallant, 2007) including random forest classification (Corcoran et al., 2013) with some complex models drawing from numerous data layers to discriminate among wetland types (Wright \& Gallant, 2007). As a result, it is not surprising that many studies have been devoted entirely to comparing the utility of these different methods (Dingle-Robertson \& King, 2011; Duro et al., 2012; Harken \& Sugumaran, 2005; Shanmugam et al., 2006) with no general consensus reached on a universal methodology. Similarly, the use of ancillary data in improving wetland mapping accuracy has been demonstrated by the inclusion of LIDAR (Hopkinson et al., 2005) and RADAR data (Grenier et al., 2007) to characterize vegetation height, time series image data for wetland boundary and change detection (Davranche et al., 2010; Johnston \& Barson, 1993), and passive microwave data to map flooded areas (Prigent et al., 2001). Understandably, the process of mapping complex and variable ecosystems such as wetlands have led to equally complex approaches.

This paper focusses instead on the variability in landscapes where wetlands are found and applies a parsimonious approach to mapping these features across each variable scene using high spatial resolution 4-band multispectral imagery from WorldView2 and GeoEye1. Here, we employ a constant GEOBIA supervised-classification approach to wetland landcover mapping across three landscapes varying in disturbance from human activity representing a semi-natural park, agricultural, and urban landscape, to determine the robustness of this method across scenes of varying heterogeneity and composition.

\section{Study Area}

Three study sites were selected and categorized as natural, agricultural, and urban. As most natural areas have undergone some level of alteration or disturbance, we define the natural landscape and cover types based on criteria adapted from Fahrig et al. (2011) as areas where (1) most primary production is not consumed by humans, either directly or indirectly, (2) the main species of the cover type has an evolutionary or long-term association with that area, and (3) the frequency and intensity of anthropogenic disturbances are low relative to those in agricultural and urban regions. Study sites were further categorized based on population density with an urban area defined as an 
157 area of over 400 people $/ \mathrm{km}^{2}$, a rural-agricultural area of less than 400 people $/ \mathrm{km}^{2}$, and a natural 158 site with no permanent human population which was represented by a relatively undisturbed 159 landscape (http://www.statcan.gc.ca/subjects-sujets/standard-norme/sgc-cgt/notice-avis/sgc-cgt-06160 eng.htm).

161 The natural study site is located in the northeast corner of Algonquin Provincial Park (Ontario, 162 Canada), hereafter referred to as the park site, which represents a protected and relatively 163 undisturbed landscape (Figure 1a). The park was established in 1893 and encompasses 7,630 km² 164 which includes approximately 340 ha of wetlands of all classes as defined by the Canadian 165 Wetlands Classification System (NWWG, 1997). Logging activity occurs in the study area as well 166 as recreational use by park visitors, though the study site is located in a less heavily visited section. 167 The agricultural site is in the County of Brant (Ontario, Canada) which sits within the Grand River 168 watershed and is located approximately $130 \mathrm{~km}$ west of Toronto, supporting a population of 35,000 169 people (Figure 1b). Provincial and private roads bisect the agriculturally dominated landscape and 170 surround the Oakland Swamp, an 890 ha wetland of provincial significance. Several smaller 171 wetlands of variable size and shape are also distributed throughout the study area. The urban study 172 site encompasses the eastern portion of Toronto and the adjacent city of Pickering (Figure 1c). 173 Toronto is the largest city in Canada and supports a population of 2.79 million people, and a 174 greater Toronto area (GTA) population of 5.5 million. The study site includes the Rouge Urban 175 National Park, a federally-governed urban recreation area covering roughly 6300 ha and bounded 176 along its western and eastern border by dense urban development including roadways that cross 177 over and through the park interior. A dense pocket of wetlands can be found in the southernmost 178 portion of the park near the Lake, and several smaller wetlands are also scattered within the 179 northern portion of the park and in the adjacent urban and rural regions, including several recently 180 restored wetlands. This urban park receives thousands of visitors annually and its interior is 181 bisected with pedestrian and bike pathways. 

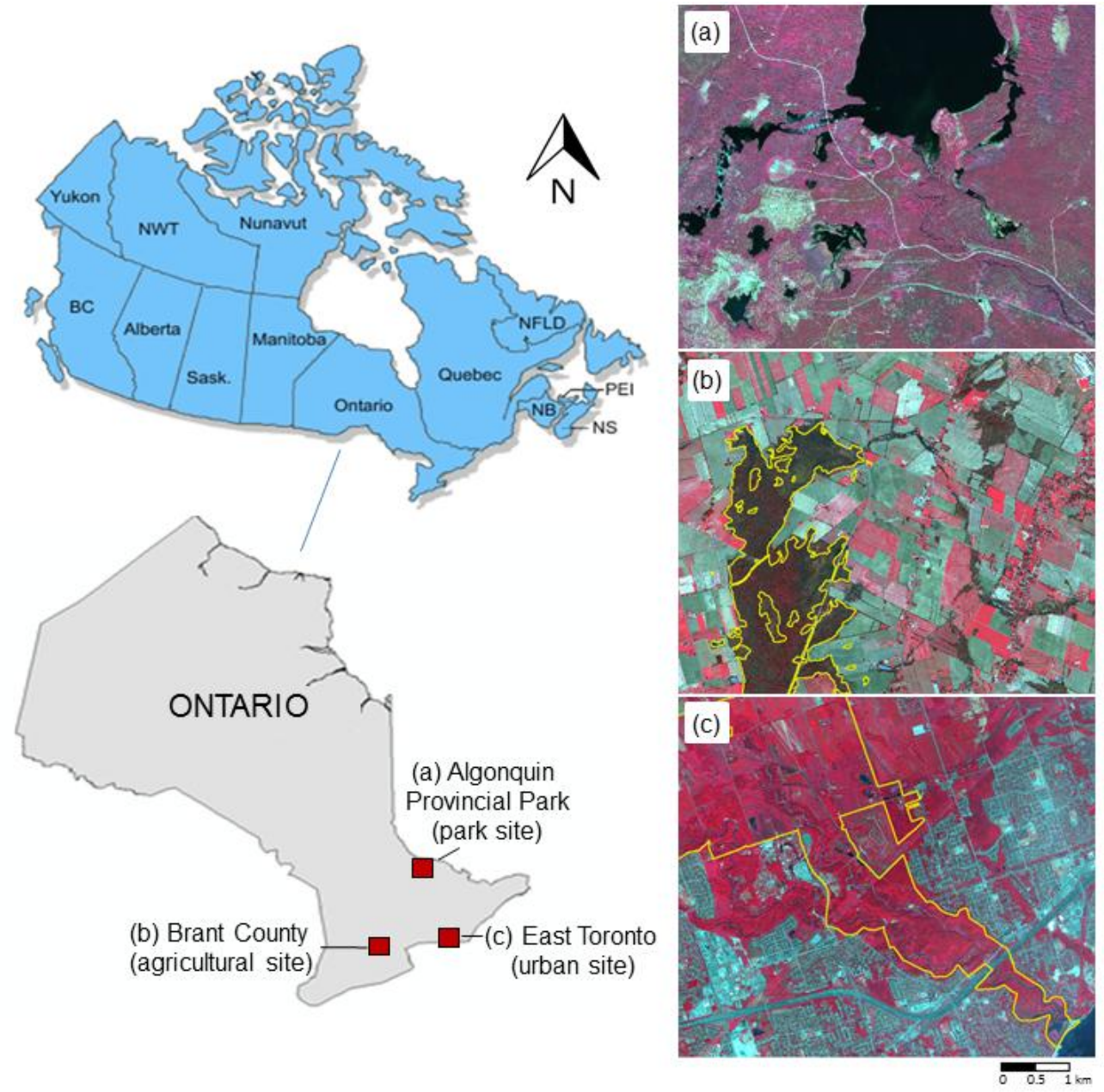

Figure 1. Study areas across southern Ontario, Canada in (a) Algonquin Provincial park site, (b) Brant County agricultural site, and (c) east Toronto urban site (satellite images from GeoEye1 [a,b], and WorldView2 [c] shown in false colour RGB=NIR-red-green). Yellow polygons indicate the provincially significant Oakland swamp [b], and the boundaries of the Rouge Urban National Park [c].

\section{Data and Methods}

190 We employed a multi-scale GEOBIA approach to segment images, which were then classified 191 using a supervised nearest neighbour method (Figure 2). Multiple input layers were utilised during 192 image segmentation with both qualitative and quantitative measures used to select and evaluate the resulting image objects. 


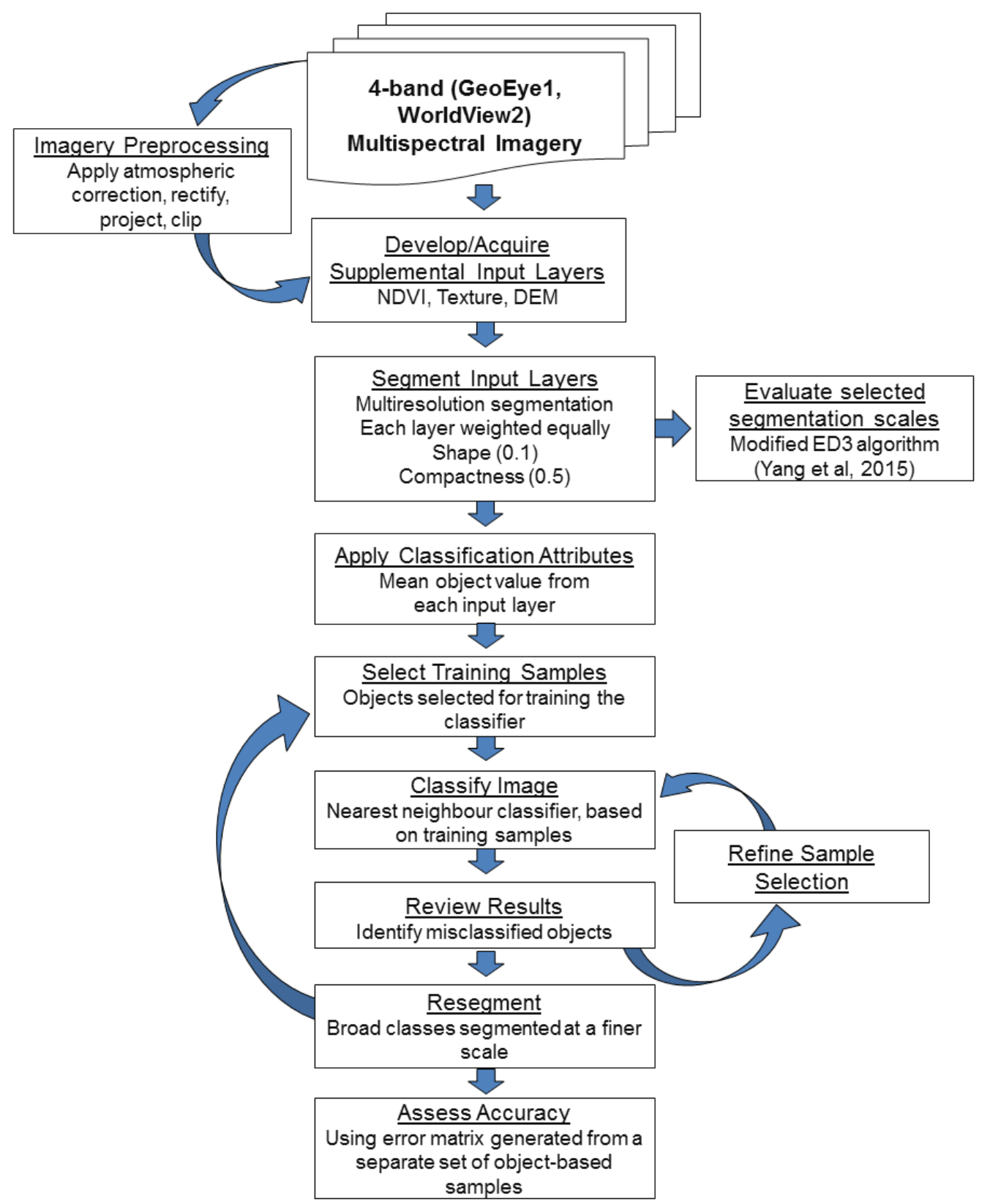

Figure 2. Process framework for segmentation and classification of wetland landscapes 
198 High spatial resolution data from WorldView2 and GeoEye1 sensors were acquired over each

Table 1. Satellite Image data information

\begin{tabular}{|c|c|c|c|}
\hline Sites & $\begin{array}{l}\begin{array}{l}\text { Rouge Park } \\
\text { (urban site) }\end{array} \\
\end{array}$ & $\begin{array}{c}\text { Algonquin Park } \\
\text { (natural site) }\end{array}$ & $\begin{array}{c}\text { Brant County } \\
\text { (rural site) }\end{array}$ \\
\hline Acquisition date & 25 July 2012 & 25 May 2013 & 9 April 2012 \\
\hline Sensor & WorldView2 & \multicolumn{2}{|c|}{ GeoEye 1} \\
\hline Spatial Resolution (m) & \multicolumn{3}{|c|}{$2 \mathrm{~m}$ multispectral } \\
\hline Spectral Resolution & $\begin{array}{c}\text { Blue }(450-510 \mathrm{~nm}) \\
\text { Green }(510-580 \mathrm{~nm}) \\
\text { Red }(630-690 \mathrm{~nm}) \\
\text { Near infrared }(770-895 \mathrm{~nm})\end{array}$ & $\begin{array}{r}\text { Blue }(45 \\
\text { Green }(5 \\
\text { Red }(65 \\
\text { Near infrare } \\
\end{array}$ & $\begin{array}{l}\text { m) } \\
\text { im) } \\
\text { n) } \\
20 \mathrm{~nm})\end{array}$ \\
\hline
\end{tabular}

211 All images were radiometrically normalized using the ATCOR module implemented through PCI

212 Geomatica (PCI Geomatica, 2014), and projected to the Universal Transverse Mercator projection

213 datum (NAD83, UTM Zone 17). The scenes were georeferenced to a root mean squared error of

214 less than 2 pixels using a $1^{\text {st }}$ order polynomial transformation and nearest neighbour resampling

215 method, and clipped to the same extent using ArcGIS version 10.2 (Environmental Systems

216 Research Institute, Redlands, CA, USA). The panchromatic layer was not used, as it increased

217 processing time to unrealistic lengths. 
219 Seven features (or input layers) were used for image segmentation including four multispectral

220 layers (blue, green, red, and near infrared), a DEM layer, an NDVI (normalized difference

221 vegetation index) layer, and a standard deviation texture layer.

222 A 10-m digital elevation model (DEM) was acquired from the Ontario Ministry of Natural

223 Resources (Figure 3a) which was interpolated from an DTM (digital terrain model), a contour map,

224 spot height data, and a water virtual flow map to a $\pm 10 \mathrm{~m}$ vertical precision. The DEM for each

225 image scene was resampled to $2 \mathrm{~m}$ to match the resolution of the other input layers. Resampling

226 the DEM did not provide any additional information but ensured continuity in pixel size across all

227 input layers and avoided a coarser resolution affecting the boundaries of image objects. Elevation

228 was included as an input layer because wetlands and water bodies are known to sit topographically

229 low in the landscape due to their close association with ground water and surface run-off (Mitsch

$230 \&$ Gosselink, 2000). Other elevation-related input layers such as slope and aspect were originally

231 included, but were discarded as they did not contribute any additional information.

232 Texture information refers to the spatial variation in the spectral brightness of a digital image, and

233 has a high potential for revealing differences between classes in remotely sensed imagery

234 (Berberoğlu et al., 2007). Texture measure can also be derived directly from satellite imagery, and

235 do not require the acquisition of additional data. For this study we created a first-order texture layer

236 (Figure $3 \mathrm{~b}$ ) based on the standard deviation within a 3 pixel by 3 pixel moving window. A 3 by 3

237 window was selected as they most likely to cross spatial resolutions with the least areal effects

238 (Ryherd \& Woodcock, 1996).

239 NDVI is a well-established indicator of live green vegetation (Rouse et al, 1974). An NDVI layer 240 was thus created from the red and near infrared bands of the multispectral data to separate water 241 from dry land, and delineating wetland boundaries (Ozesmi \& Bauer, 2002) (Figure 3c). Other 242 input layers such as slope and aspect were originally included, but were discarded as they did not 243 contribute any additional information. All final image layers were weighted equally in the 244 segmentation process. 

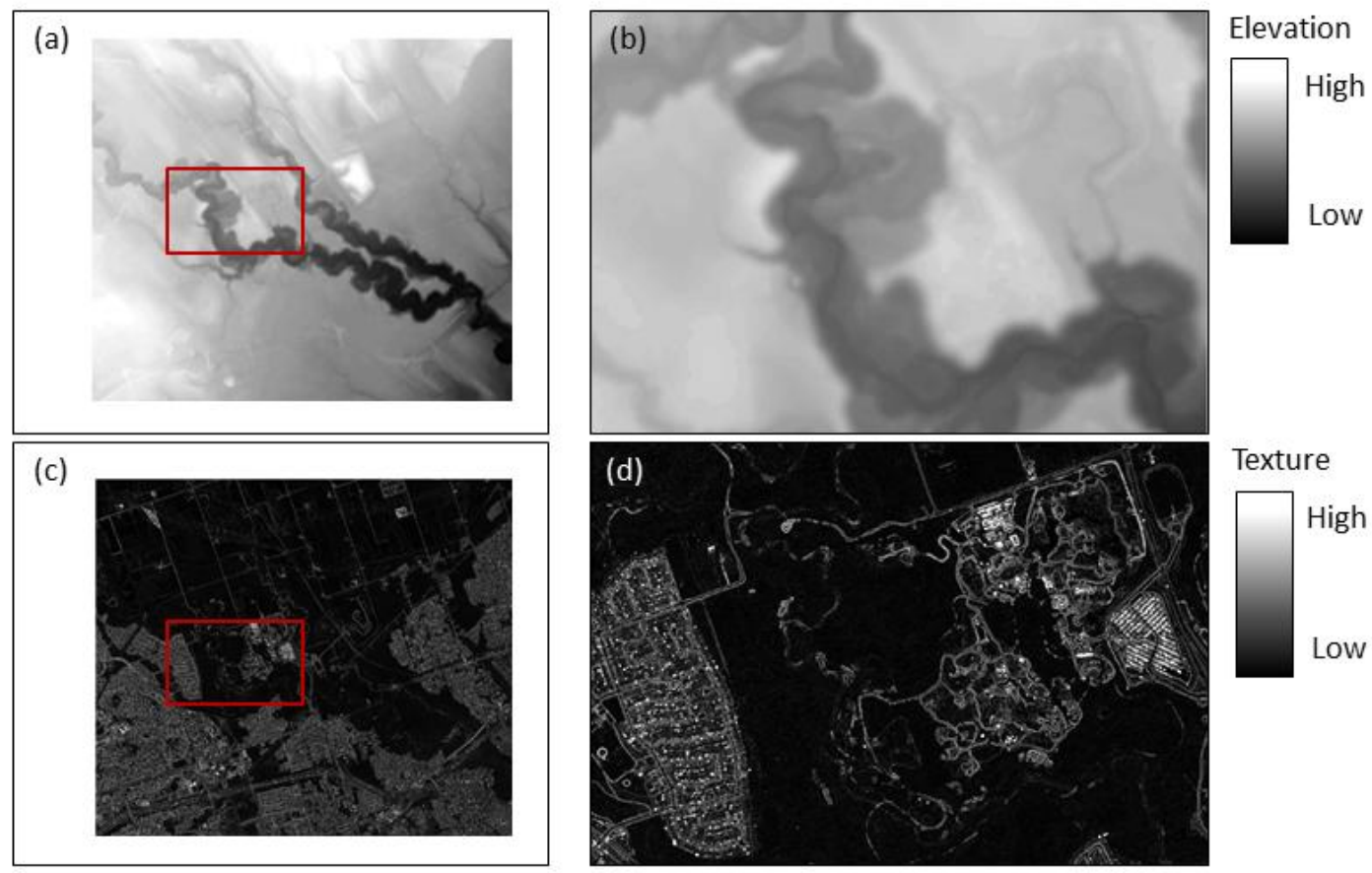

\section{Texture}
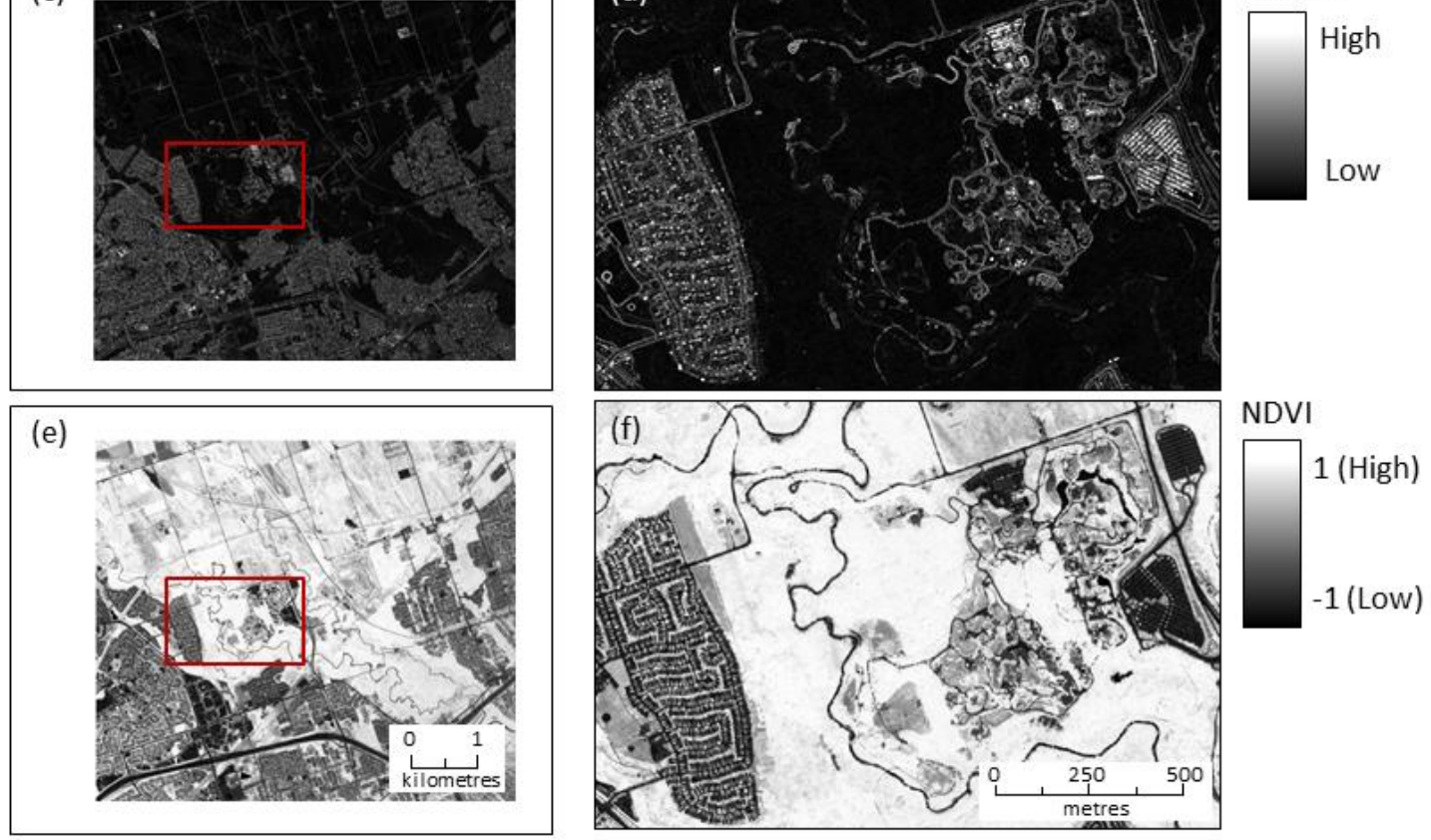

Figure 3. Subset of input layers from the urban site of eastern Toronto showing (a) a digital elevation model layer, (b) a standard deviation texture layer, and (c) an NDVI layer

252 Segmentation is a key aspect of GEOBIA relating to the ultimate quality of the final classification 253 (Baatz et al., 2008) and its optimal result is a scene segmented into objects that reflect real-world 254 features of interest. This study employed a multiresolution segmentation algorithm based on the 255 fractal net evolution approach (FNEA; Baatz \& Schape, 2000) implemented in Definiens 256 Developer 7.0 (Munich, Germany; Definiens, 2008, formerly eCognition). 
Three key segmentation parameters - shape $\left(\mathrm{S}_{\mathrm{sh}}\right)$, compactness $\left(\mathrm{s}_{\mathrm{cm}}\right)$, and scale $\left(\mathrm{S}_{\mathrm{sc}}\right)$ - control the

258 size, shape and spectral variation of segmented image objects. Shape parameters were set to 0.1 to

259 place greater emphasis on pixel values of input layers rather than shape, and compactness was set

260 to 0.5 to balance both compactness and smoothness of object boundaries equally. The most critical

261 step is the selection of the scale parameter which controls the size of the image objects. The scale

262 parameter sets a threshold of homogeneity which determines how many neighbouring pixels can be

263 merged together to form an image object (Benz et al., 2004).

264 In this paper, we applied a multi-scaled segmentation approach that utilized three levels of scale 265 parameterization to capture different landcover classes (Figure 4). Dominant landcover classes that 266 covered the majority of the scene were segmented at the coarse level, while remaining classes were 267 delineated at the medium level. Entire wetlands were segmented and defined as objects at the mid268 range scale, and further segmented at the finest scale level to delineate components within 269 wetlands and classify these as marsh, swamp, fen or bog. These smaller (child) objects retain links 270 to their larger (parent) class which employs a true multi-scale approach through applying vertical 271 constraints in segmentation and classification. Classification for specific landcover classes was 272 thus completed at each scale level, with remaining unclassified objects undergoing further 273 segmentation, followed by classification. A thematic road network layer was available for each 274 scene and was used in the segmentation process.

275 We first employed a qualitative visual approach to select the scale parameter at each level (coarse, 276 medium, and fine). At the medium and fine segmentation level this ensured that the optimal scale 277 parameter for wetlands was selected by drawing upon knowledge of the study areas, and based on 278 the premise that the human eye is best capable of interpreting and recognizing complex patterns in 279 conjunction with neighbourhood context (Benz et al., 2004; Myint et al., 2011). This approach is 280 especially fitting for wetlands that can be highly variable in both size and shape. Scale values 281 ranging from 5 to 250 with an interval of 5 were evaluated for each image and final scale selection 282 was guided by field knowledge, thematic maps and aerial imagery. 


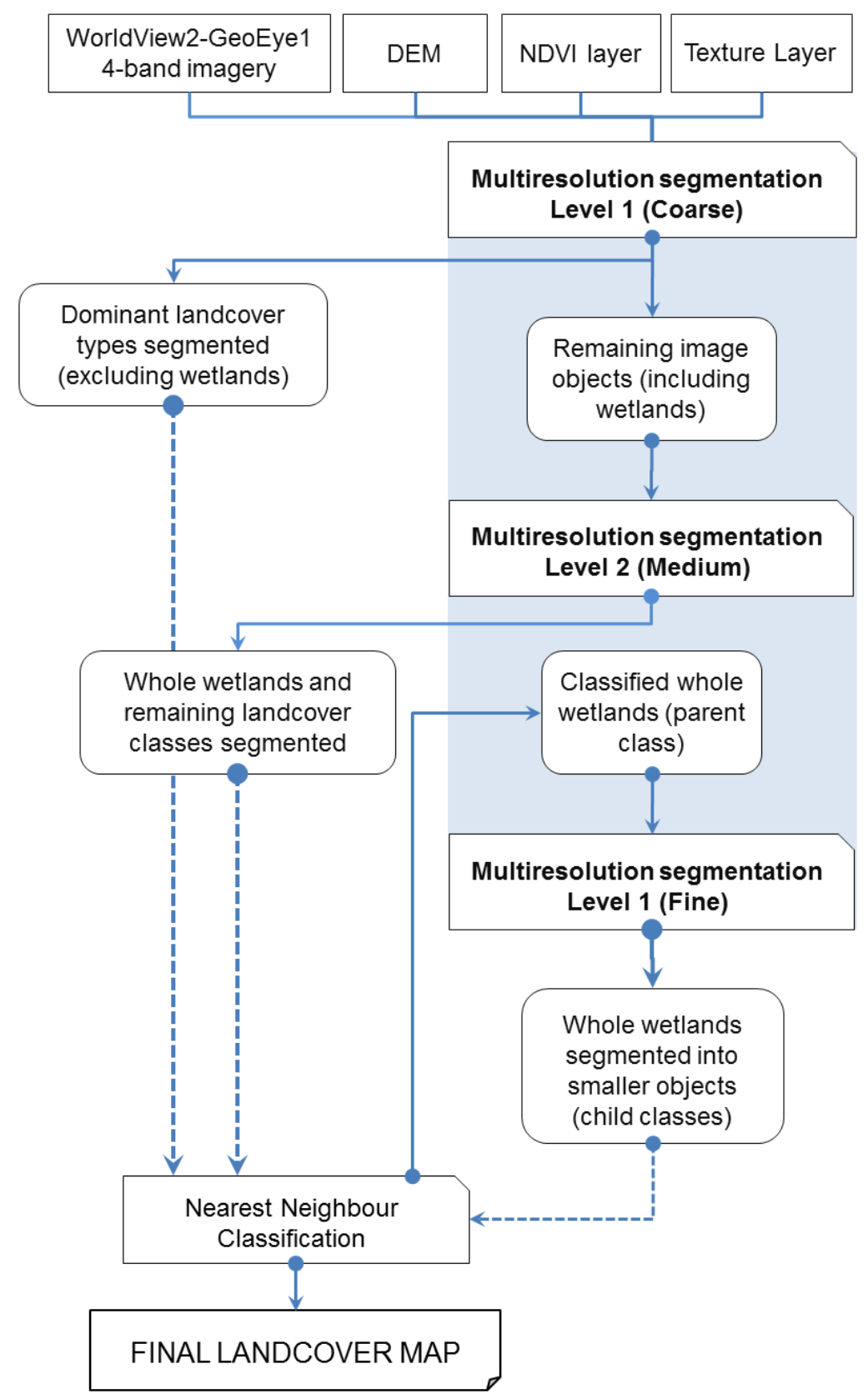

Figure 4. Multi-scale segmentation process used to segment images at three levels, with a hierarchical parent-child relationship developed between wetlands and within wetland components at the medium (level 2) and fine (level 1) scale. 
This selection was then quantitatively evaluated using the modified ED3 discrepancy measure of Yang, He, and Weng (2015) based on global geometric and arithmetic relationships (e.g. overunder segmentation) between hand-digitized reference polygons and corresponding segments produced by the multiresolution segmentation algorithm. This multi-band scale parameter evaluation method allows identification of multiple appropriate scale parameters, where a candidate segment will be labelled as the corresponding segment of a reference polygon only when the overlapping area is over $50 \%$. Results are normalized between zero and 0.71 , with lower values indicating a higher segmentation quality (Yang et al., 2015). Multiresolution segmentation results between scale values of 5 to 200 (intervals of 5) were compared to a set of manually delineated reference polygons at each scale level (coarse, medium, fine), and for each image. A total of 30 reference polygons per scale level were used in the analysis. There are multiple quantitative and automated approaches to selecting the scale parameter including automated parameterisation using the potential of local variance to detect scale transitions (Drăguţ et al., 2014; Drăguţ et al., 2010), supervised methods that use various indices to describe the discrepancy between reference polygons and corresponding image objects (Clinton et al., 2010; Liu et al., 2012), and a comparison index using both topological and geometric object metrics (Moller et al 2007). However, there is no perfect algorithm that is appropriate for all images (Munoz et al, 2003) and a certain element of trial, error, and repetition is inherent to the overall process of scale selection and evaluation.

\section{Sample selection and classification}

Our final classification scheme was based on the land cover and land use classification system developed by Anderson et al. (1976). Specifically, we aimed to classify our study areas into following classes: agricultural land, barren land, forested upland, herbaceous upland, urban matrix, water, and wetland (Table 2). Wetlands were broadly defined as land that is saturated with water for a period of time sufficient to promote wetland or aquatic processes resulting in characteristics such as poorly drained soils, hydrophytic vegetation, and other biological activity adapted to wet environments (NWWG, 1997). According to the Canadian Wetland Classification System (NWWG, 1997) wetlands were further classified as marsh, swamp, bog, or fen (Table 3). All wetland classes were found in the park study site, while only marshes and swamps were found in the agricultural and urban locales. 

wetland classification system (National Wetlands Working Group, 1997)

\begin{tabular}{ll}
\hline Class & Description \\
\hline Agricultural Land & $\begin{array}{l}\text { Land used primarily for production of food and fiber (e.g., Row crops, } \\
\text { bare (idle) fields, shaded crops; groves; orchards) }\end{array}$ \\
Barren Land & $\begin{array}{l}\text { Land of limited ability to support life; less than one-third of the area has } \\
\text { vegetation or other cover (e.g., sands, rocks, thin soil) }\end{array}$ \\
Forested Upland & Closed canopy deciduous, coniferous, or mixed forests \\
Herbaceous Upland & $\begin{array}{l}\text { Land where vegetation is dominated by a mix of grasses, grass-like plants, } \\
\text { forbs, shrubs or bush; either naturally-occurring or modified (e.g. old } \\
\text { fields, roadside vegetation, meadows, mixed composition short vegetation } \\
\text { upland) }\end{array}$ \\
Urban or Built & $\begin{array}{l}\text { Areas of intensive use with much of the land covered by man-made } \\
\text { structures (e.g., residential, commercial, industrial, utility, and } \\
\text { transportation sites such as those found in cities, towns, rural communities } \\
\text { and strip developments) }\end{array}$ \\
Watrix & $\begin{array}{l}\text { All areas that are persistently water-covered (e.g., lakes, reservoirs, } \\
\text { streams, bays, estuaries) }\end{array}$ \\
Wetland & Bog, fen (or wet meadow), swamp, marsh, shallow open water \\
\hline
\end{tabular}

\begin{tabular}{ll} 
Class & Description \\
\hline Bog & $\begin{array}{l}\text { A peat landform, raised or level with the surrounding terrain and isolated } \\
\text { from runoff and groundwater, receiving water primarily from } \\
\text { precipitation, fog, and snowmelt. Water table sits at or slightly below the } \\
\text { bog surface. Treed or treeless, and usually covered with Sphagnum spp. } \\
\text { and shrubs, or woody remains of shrubs. }\end{array}$ \\
A type of peatland which receives both surface and groundwater flow \\
due to its topographic position which is level with the surface of the fen \\
(+/- a few centimetres). Vegetation can include graminoids, bryophytes, \\
shrubs, and also trees (in drier fens). \\
A shallow water wetland with water levels that can fluctuate daily, \\
Marsh \\
annually, or seasonally resulting in highly variable hydrology. Receives \\
water from the surrounding catchment as well as precipitation. Marsh
\end{tabular}


vegetation is comprised of emergent aquatic macrophytes such as graminoids (e.g. rushes, reeds, sedges), floating-leaved species (e.g. lilies) and submergent species (e.g. water milfoil). Marsh plant communities are seasonal and dynamic, often shifting with water levels.

Swamp Forested or wooded wetland, dominated by minerotrophic groundwater and a water table below the ground surface of the swamp for the majority of the year. Vegetation dominated by coniferous or deciduous trees or tall shrubs (generally over $30 \%$ ).

326 Sample selection - Training sample objects were selected using aerial photographs, thematic maps 327 and ground truth data collected during field campaigns. A minimum of 50 training sample objects 328 were chosen for each class, with some exceptions for classes which only covered a small 329 proportion of the scene. An advantage of the multi-scale approach is the ability to adequately 330 sample rarer classes such as wetlands by segmenting these landforms into smaller image objects. A 331 random stratified sampling strategy was employed for sample selection with additional samples 332 collected over rare classes as needed. Sample image objects for wetlands were grouped into classes 333 as defined by the Canadian Wetland Classification System. In some cases, a dominant class such as 334 'marsh' was further separated into emergent marsh and wet meadow in order to capture the spectral and textural variation in these heterogeneous marsh communities. These groups were later merged into one marsh class for comparison across landscapes.

337 Nearest Neighbour Classifier - A non-parametric nearest neighbour classifier was used to place 338 image objects into defined landcover classes. This iterative process involved selecting training 339 samples, comparing sample attributes, and refining training samples until a satisfactory result was 340 achieved. The nearest neighbour classifier is advantageous when image data are composed of 341 spectrally similar classes that are not well separated using a few features or just one feature 342 (Definiens, 2008) and also when training sample sizes may be uneven (Myint et al., 2011; Yu et 343 al., 2006). The mean feature values of pixels in each object (calculated from the input layers), were 344 used to quantify separation distance between classes. The nearest neighbour, or k-NN approach as 345 it is often called, is a simple yet efficient classification algorithm that has been shown to perform as 346 well as more complicated methods such as support vector machines (SVM) under constant 347 conditions (Im et al., 2008). There are many attributes that can be used to inform the nearest 348 neighbour classifier; however, the contribution of each will vary and constraints such as processing 349 time will dictate the maximum number. We developed a parsimonious model based upon the mean 
object value for each input layer, in order to maintain a realistic processing time and an efficient model that can be compared across landscapes.

\section{Accuracy assessment}

354 A minimum of 35 independently selected samples were used for accuracy assessment. Sample 355 selection was based upon very high resolution (VHR) aerial photographs over each site, reference 356 thematic maps, and ground truth data collected in June 2011 from each study area. Validation and

357 training samples did not overlap. Accuracy was assessed based on the error matrix and associated 358 statistics, namely overall accuracy, kappa statistic and producer's accuracy (1 - errors of omission) and user's accuracy (1 - errors of commission).

\section{Results}

Multi-scale segmentation

362 Final scale values selected through visual assessment varied between study areas (Table 4). 363 Dominant landcover classes of mixed forest (park site), crop field (agricultural site), and urban 364 matrix (urban site) were most accurately delineated at a scale of 125, 200, and 75 respectively. 365 Boundaries were generally clearly defined with minimal absorption of smaller classes. Similarly, 366 whole wetland boundaries were generally well defined and often included greater spatial detail 367 than reference thematic maps, although specific depiction varied across each landscape. Medium 368 level scale values of 40, 60, and 50 were selected at the park, agricultural, and urban site respectively. Whole wetlands were further segmented at the finest scale level (20 [park], 10

370 [agricultural], 15 [urban]) to further classify these objects into marsh, swamp, bog, fen, or water. 371 This parent-child relationship maintained a hierarchical constraint which limited classification of 372 the five wetland classes to only those objects defined earlier as wetlands. Scales values of 20 373 (park), 10 (agricultural) and 15 (urban) were selected at the finest level. Segmentation scales varied 374 across all scenes, and no segmentation scale mirrored those of the other sites at any level. 
Table 4. Hierarchical segmentation scale for each study site and corresponding landcover class

\begin{tabular}{clclcc}
\hline \multicolumn{2}{c}{ Park Landscape } & \multicolumn{2}{c}{ Agricultural Landscape } & \multicolumn{2}{c}{ Urban Landscape } \\
\hline Scale & Target Landcover & Scale & Target Landcover & Scale & Target Landcover \\
\hline 125 & $\begin{array}{l}\text { Forested upland, } \\
\text { water }\end{array}$ & 200 & Agricultural fields & 75 & $\begin{array}{c}\text { Urban matrix, } \\
\text { agricultural fields }\end{array}$ \\
\hline 40 & $\begin{array}{l}\text { Wetland, barren } \\
\text { land, herbaceous } \\
\text { upland }\end{array}$ & 60 & $\begin{array}{l}\text { Wetlands, water, } \\
\text { urban matrix, } \\
\text { meadow }\end{array}$ & 50 & $\begin{array}{c}\text { Wetlands, water, forests, } \\
\text { herbaceous upland, } \\
\text { barren land }\end{array}$ \\
Wetland classes & 10 & wetland classes & 15 & Wetland classes \\
\hline
\end{tabular}

379

380 Modified ED3 results showed a consistent positive evaluation for all scale parameters selected by

381 visual assessment (Figure 5). ED3 results range from 0 to 0.71 with lower values corresponding to

382 better quality segments that more closely match with reference polygons (Yang et al, 2015). In the

383 corresponding graphs, the selected scales fell within the lowest dip in the data points, which

384 characterises scale parameters with the greater fitness in matching with reference polygons (Yang

385 et al, 2015). Across all scale levels (coarse, medium, fine) and image scenes (park, agricultural,

386 urban), scale values selected through visual assessment fell within this region indicating a robust

387 selection. Interestingly, at the coarse (first) level, results across all three scenes do not demonstrate

388 a pronounced trough but rather a gradual descent in values indicating that several scale values are

389 appropriate at this level. 

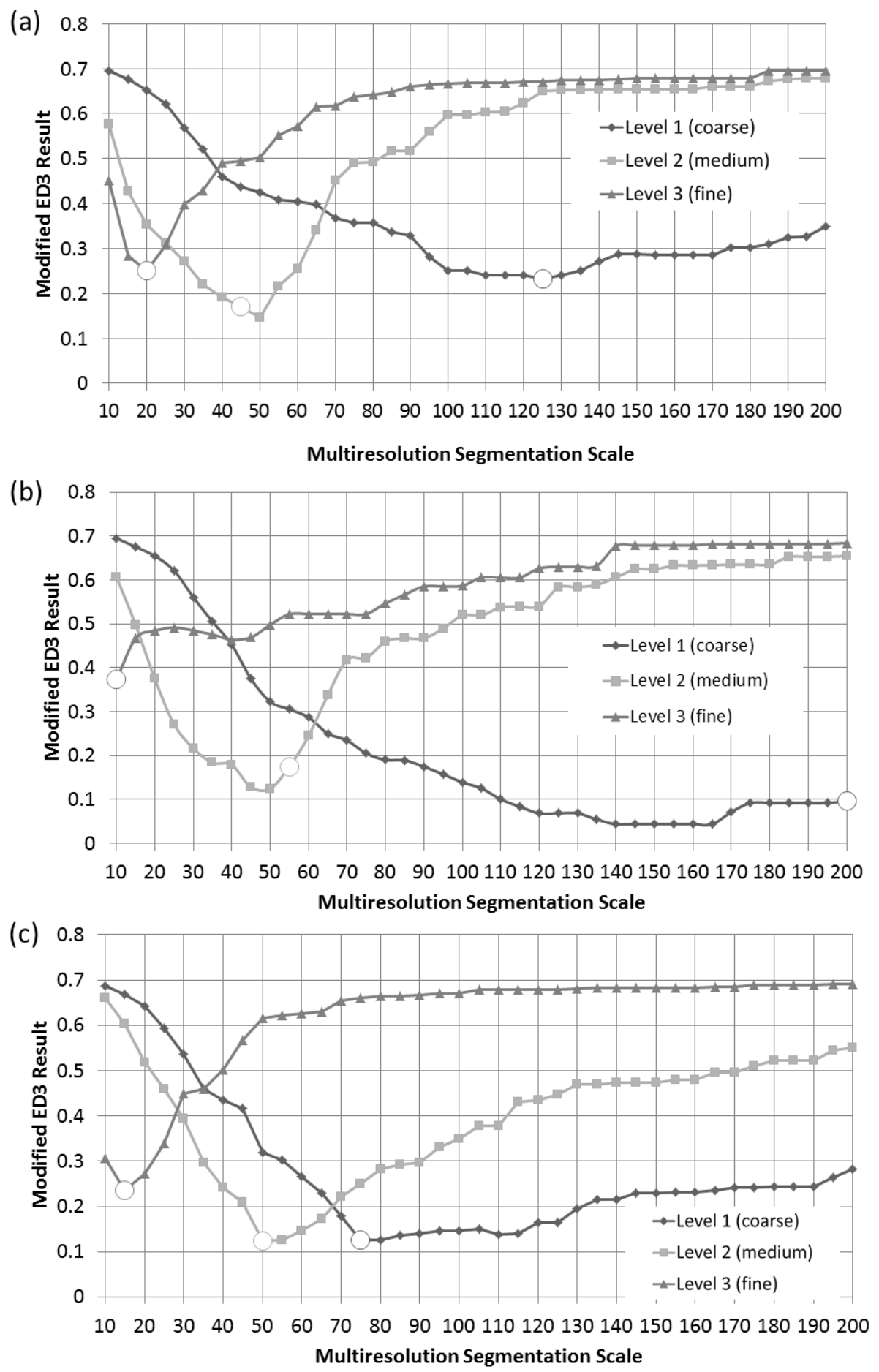

Figure 5. Quantitative evaluation of selected scale parameter with the modified ED3 algorithm at the coarse (diamond), medium (square), and fine (triangle) levels for the (a) Algonquin park site, (b) Brant county agricultural site and (c) east Toronto urban site.

401 Hollow circles denote the scale value selected through visual assessment. 
The contribution of supporting input layers (DEM, NDVI, texture) improved overall segmentation results across all scenes. At the coarse level, we found the inclusion of the NDVI layer improved delineation of vegetated boundaries. For example in the agricultural scene, NDVI data improved crop field segments such that object boundaries more closely followed the outer edges of each field (Figure 6). At the medium level, elevation data from the DEM resulted in improved segmentation of wetland boundaries (Figure 7). Texture information was useful at the finest scale level for segmenting medium scale wetland objects (Figure 8). The inclusion of this layer resulted in larger image objects more representative of distinct vegetation communities.
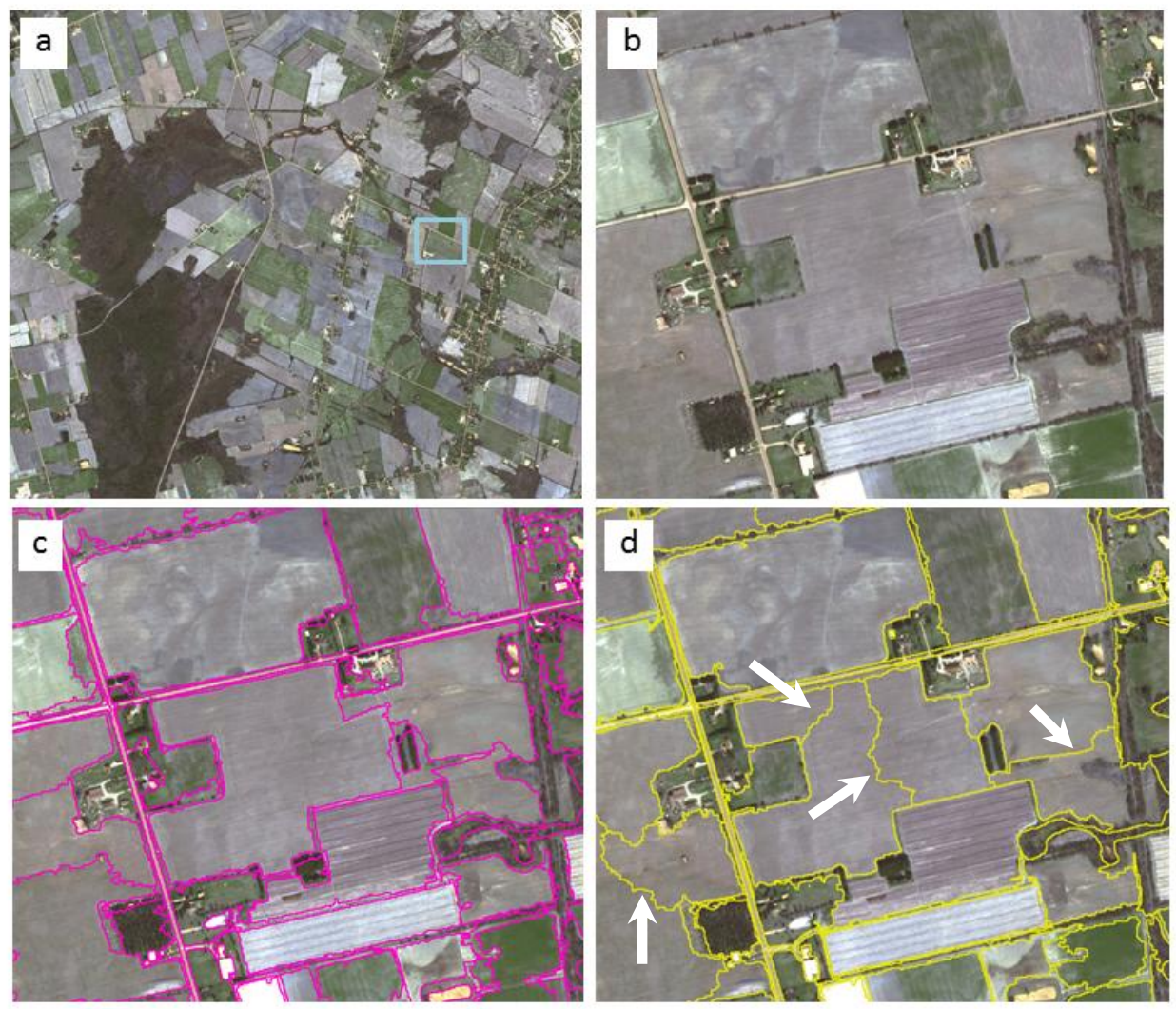

411 Figure 6. Comparison of coarse level segmentation results over the Brant County agricultural scene 412 and subset (a, b), at scale 200 using all seven input layers (c), and at the same scale 200 with the 413 NDVI layer excluded (d). White arrows in (d) show locations of over-segmentation that do not 414 correspond with crop field boundaries. 

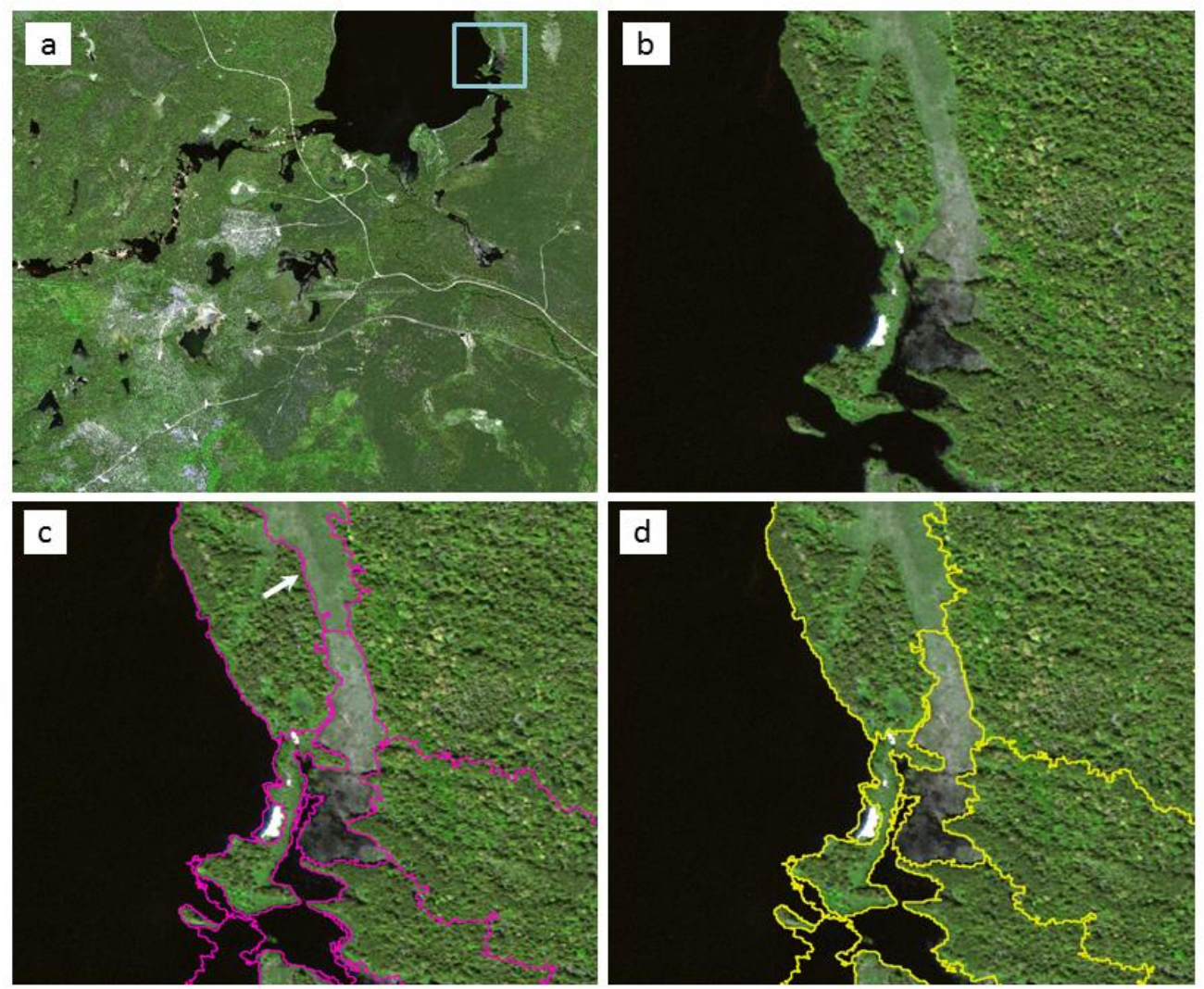

416 Figure 7. Comparison of medium level segmentation results over the Algonquin park scene (a) and 417 subset of a marsh, fen, bog complex (b) at scale 40 using all seven input layers (c), and at the same 418 scale 40 with the DEM layer excluded. White arrow shows improved segmentation of a wetland 419 boundary (to the right of the indicated line) with the inclusion of the DEM layer. 

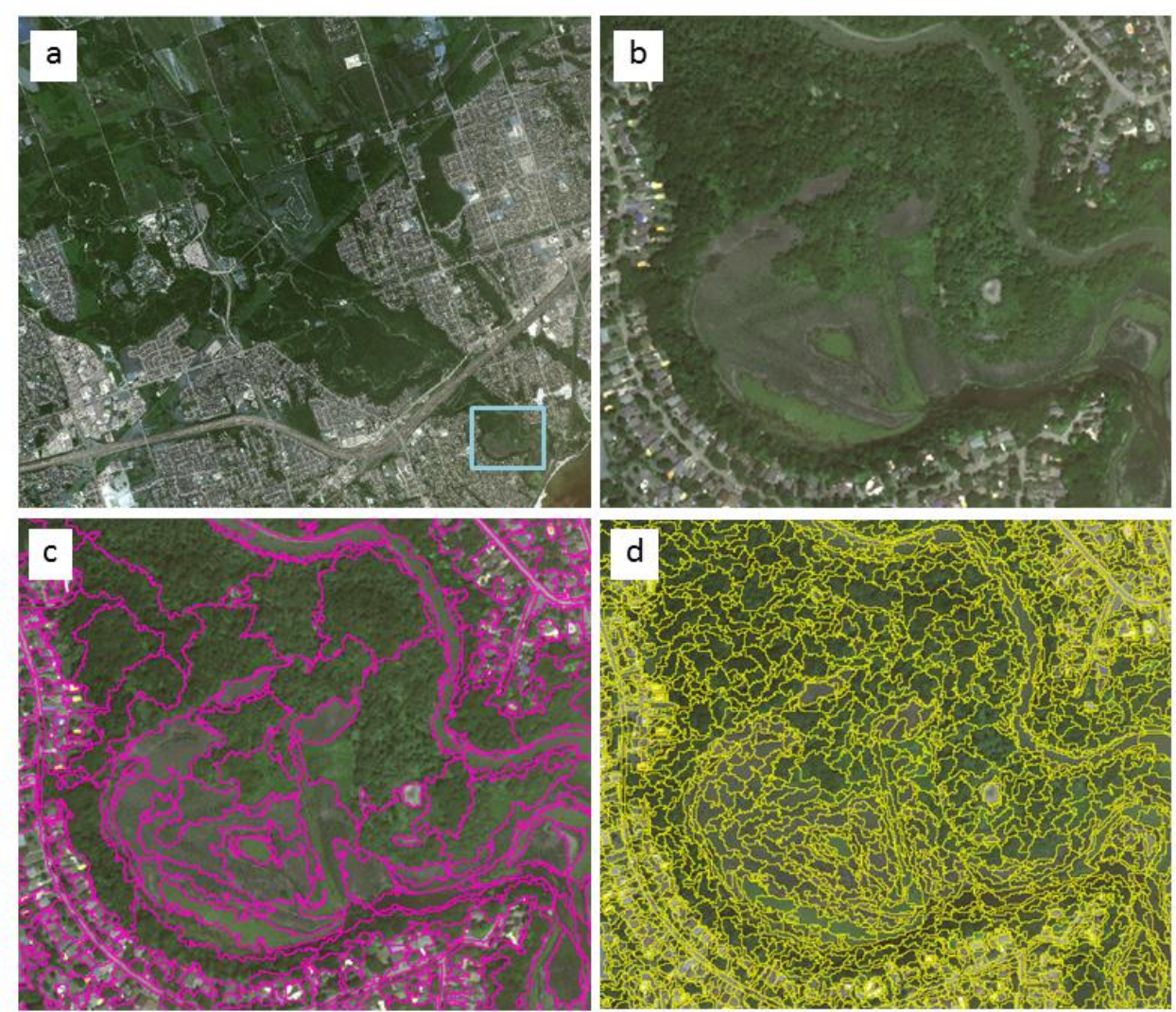

421 Figure 8. Comparison of fine level segmentation results over the east Toronto urban scene (a) and 422 subset of a marsh complex (b) showing scale 15 using all seven input layers (c), and at the same 423 scale 15 with the texture layer excluded. Note the significant over-segmentation of the texture424 excluded image.

426 A comparison with reference thematic maps indicated that the segmentation captured a greater

427 level of variation in wetland components such as floating vegetation, islands, and water (Figure 9

428 a,b,c), yet suffered from a varying degree of over and under segmentation when the swamp class

429 was present (Figure 9 d,e,f). Multispectral data from the shorter visible and near infrared

430 wavelengths did not capture information from the mid infrared water-absorbing regions, therefore

431 if standing water was not evident at the time of image acquisition, swamps could be easily

432 confused with upland forests. In both the rural and agricultural study areas, some wetlands were

433 identified that were missing from provincial reference datasets indicating that our approach is not

434 only able to capture additional wetlands, but also able to provide a greater level of detail

435 concerning within wetland variation. 

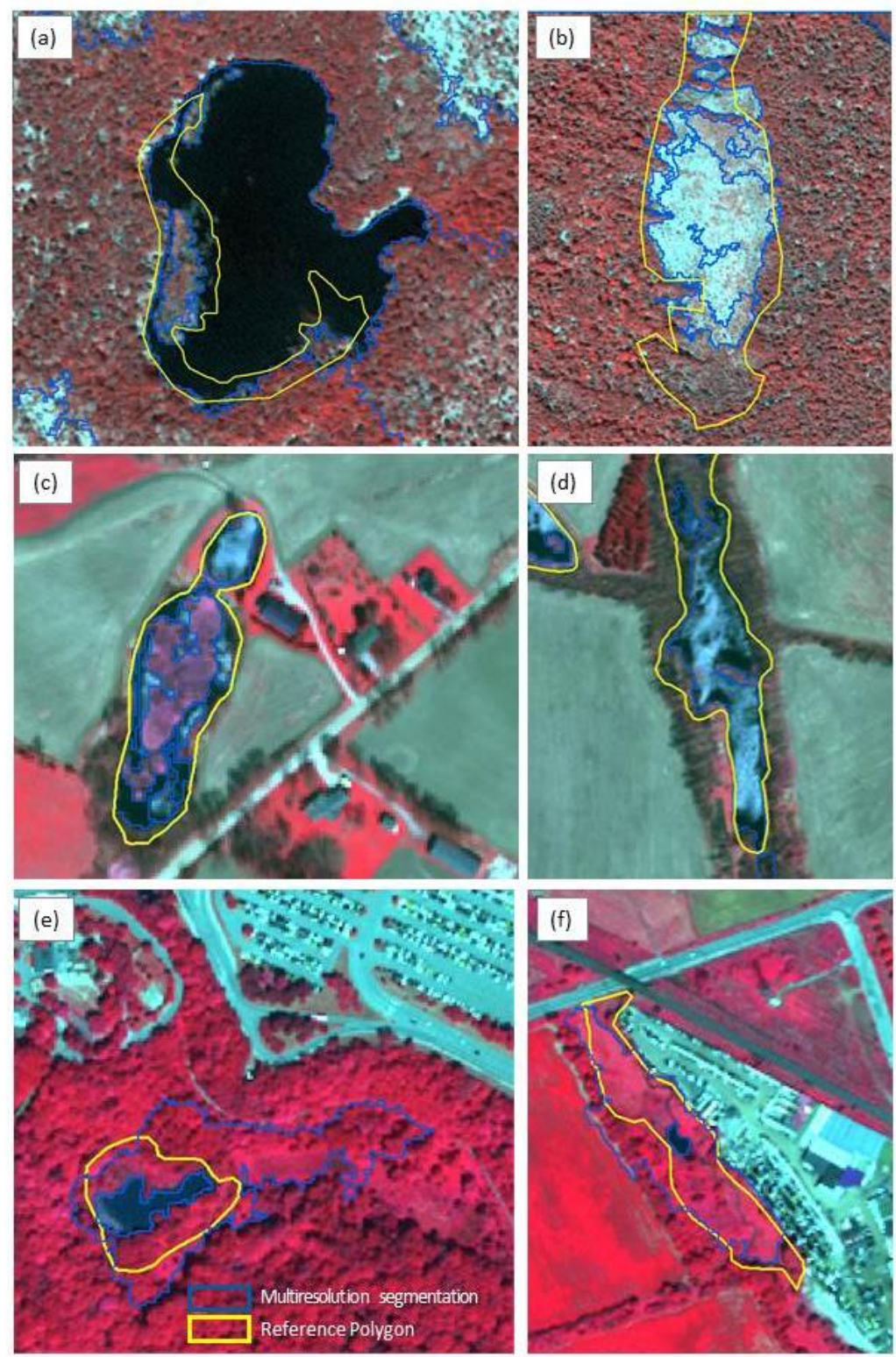

Figure 9. Sample view of wetlands enclosed by object boundaries created by the FNEA multi-scale segmentation algorithm (blue), and its corresponding reference boundary (yellow) in the natural site $(\mathrm{a}, \mathrm{b})$, rural-agricultural site $(\mathrm{c}, \mathrm{d})$ and the urban site $(\mathrm{e}, \mathrm{f})$.

442 Segmentation of non-wetland classes varied across sites. The acquisition of early spring imagery

443 resulted in better overall segmentation of dominant crop land in the agricultural scene likely due to

444 the fact that the majority of fields were bare, and borders were clearly visible. However, some 
457

\section{8}

smaller features such as hedgerows and isolated irrigation ponds suffered from absorption into these larger agricultural fields. This is attributed to variation in land-use patterns and a greater proportion of mixed vegetation classes adjacent to managed agricultural fields (see final classification maps, Figure 11). In contrast, the urban scene which also included agricultural fields suffered from a greater over and under-segmentation of crop fields, as boundaries were not as distinct in this landscape. There were no agricultural fields, or isolated ponds in the natural Algonquin Park study area and the dominant forest class was segmented with a high accuracy. While forest cover was relatively continuous across most of the scene in the natural landscape, forested uplands in the urban and rural sites were highly fragmented resulting in comparatively lower segmentation accuracy.

\section{Classification}

GeoeEye 1 and WorldView2 data were classified initially into 8 classes for the park and urban sites, and 7 classes for the agricultural site. Overall classification accuracy was the highest (kappa 0.88) over the natural Algonquin Park landscape, followed by the urban east Toronto landscape (kappa, 0.84) and lowest over the agricultural Brant County landscape (kappa 0.78). Final error matrix statistics are shown in Table 5 and final classification maps are shown in Figure 11. A different number and proportion of wetland classes (bog, fen, swamp, and marsh) were found in each landscape, and class kappa accuracy values ranged from 0.89 for the marsh class over the natural site to 0.64 for the swamp class over the rural site. Overall, forested wetlands achieved the lowest accuracy ( $0.66-0.74$ across all sites). For all study sites, water received the highest classification accuracy, followed by forests (in the natural site), agricultural fields (in the rural site), and the built/urban matrix (in the urban and rural sites).

0

71


Table 5. Error matrix statistics for landcover classes in each study site (PA = producer's accuracy, $\mathrm{UA}=$ user's accuracy)

\begin{tabular}{|c|c|c|c|c|c|c|}
\hline \multirow[b]{2}{*}{ Land cover Class } & \multicolumn{2}{|c|}{$\begin{array}{c}\text { Park Site } \\
\text { (Algonquin Park) }\end{array}$} & \multicolumn{2}{|c|}{$\begin{array}{l}\text { Agricultural Site } \\
\text { (Brant County) }\end{array}$} & \multicolumn{2}{|c|}{$\begin{array}{l}\text { Urban Site } \\
\text { (East Toronto) }\end{array}$} \\
\hline & PA & UA & $\mathbf{P A}$ & UA & PA & UA \\
\hline Marsh & 0.91 & 0.94 & 0.81 & 0.93 & 0.81 & 0.90 \\
\hline Swamp & 0.83 & 0.81 & 0.69 & 0.65 & 0.67 & 0.95 \\
\hline Fen & 0.89 & 0.83 & - & - & - & - \\
\hline Bog & 0.87 & 0.84 & - & - & - & - \\
\hline Water & 0.97 & 0.94 & 0.98 & 0.96 & 0.97 & 0.88 \\
\hline Forested Upland & 0.94 & 0.91 & 0.74 & 0.66 & 0.89 & 0.96 \\
\hline Herbaceous Upland & 0.80 & 0.82 & 0.57 & 0.50 & 0.84 & 0.74 \\
\hline Agricultural Land & - & - & 0.81 & 0.79 & 0.91 & 0.83 \\
\hline Built/Urban Matrix & - & - & 0.95 & 0.95 & 1.0 & 0.89 \\
\hline Barren Land & 0.92 & 0.95 & - & - & 0.85 & 0.85 \\
\hline Overall (kappa) & \multicolumn{2}{|c|}{$0.90(0.88)$} & \multicolumn{2}{|c|}{$0.81(0.78)$} & \multicolumn{2}{|c|}{$0.86(0.84)$} \\
\hline
\end{tabular}

Wetland-Upland Classification - Individual classes were merged into wetland (marsh, fen, bog, swamp), upland (forest, meadow, agricultural field, built, barren), and water categories in order to compare wetland accuracy amongst non-wetland classes (Table 6). Accuracy for the merged classification map was the highest for the park study site (overall accuracy 0.90, kappa 0.86), followed by the urban site (overall accuracy 0.86, kappa 0.81) with the agricultural landscape receiving the lowest accuracy (overall accuracy 0.76, kappa 0.71). Producer's accuracy was high across all study sites (> 80\%) with the exception of uplands in the agricultural landscape. Map user accuracies were generally high (> 80\%) with the exception of wetlands, and water classes in the agricultural and urban landscape (66-77\%). Across the merged classification map of the park site, only minimal errors occurred between wetland and upland classes while greater errors were found in the agricultural and urban merged maps. Focussing on wetlands, there was a high error of commission of uplands into the wetland class, and a slightly lower omission of wetland objects into the water class in the agricultural landscape. Over the urban study area wetland objects were erroneously classified as both water and upland, while only minimal errors of commission occurred. 
Table 6. Error matrix statistics for merged wetland, upland, water classes over each study site $(\mathrm{PA}=$ producer's accuracy, UA = user's accuracy).

\begin{tabular}{l|ccc|cc|cc}
\hline & \multicolumn{2}{c}{$\begin{array}{c}\text { Park Site } \\
\text { (Algonquin Park) }\end{array}$} & \multicolumn{2}{c}{$\begin{array}{c}\text { Agricultural Site } \\
\text { (Brant County) }\end{array}$} & \multicolumn{2}{c}{$\begin{array}{c}\text { Urban Site } \\
\text { (East Toronto) }\end{array}$} \\
\hline Land cover Class & PA & UA & PA & UA & PA & UA \\
\hline Wetland & 0.86 & 0.95 & 0.80 & 0.71 & 0.85 & 0.74 \\
Upland & 0.92 & 0.90 & 0.64 & 0.91 & 0.97 & 0.84 \\
Water & 0.95 & 0.97 & 1.00 & 0.66 & 0.98 & 0.77 \\
\hline Overall (kappa) & $\mathbf{0 . 9 0 ( \mathbf { 0 . 8 6 } )}$ & $\mathbf{0 . 7 6 ( \mathbf { 0 . 7 1 } )}$ & $\mathbf{0 . 8 6}(\mathbf{0 . 8 1})$ \\
\hline
\end{tabular}

498 classifier selects the most suitable attributes to classify a land cover class, we further investigated

499 the most suitable attributes used for class separation by examining overlap values between 500 classified polygons. We found that the mean object values of the red band, near infrared band and 501 NDVI provided the greatest separation between wetlands and all other classes (Figure 10). The 502 mean object value from the NDVI layer was used most frequently in discriminating wetland 503 classes from other land cover groups over the urban east Toronto site. The near-infrared layer was 504 used most frequently to separate between wetland and upland classes over the natural Algonquin 505 Park and rural Brant County sites. Texture was used to separate wetlands from built areas in the 506 rural site disproportionately more than in the natural and urban landscapes. 


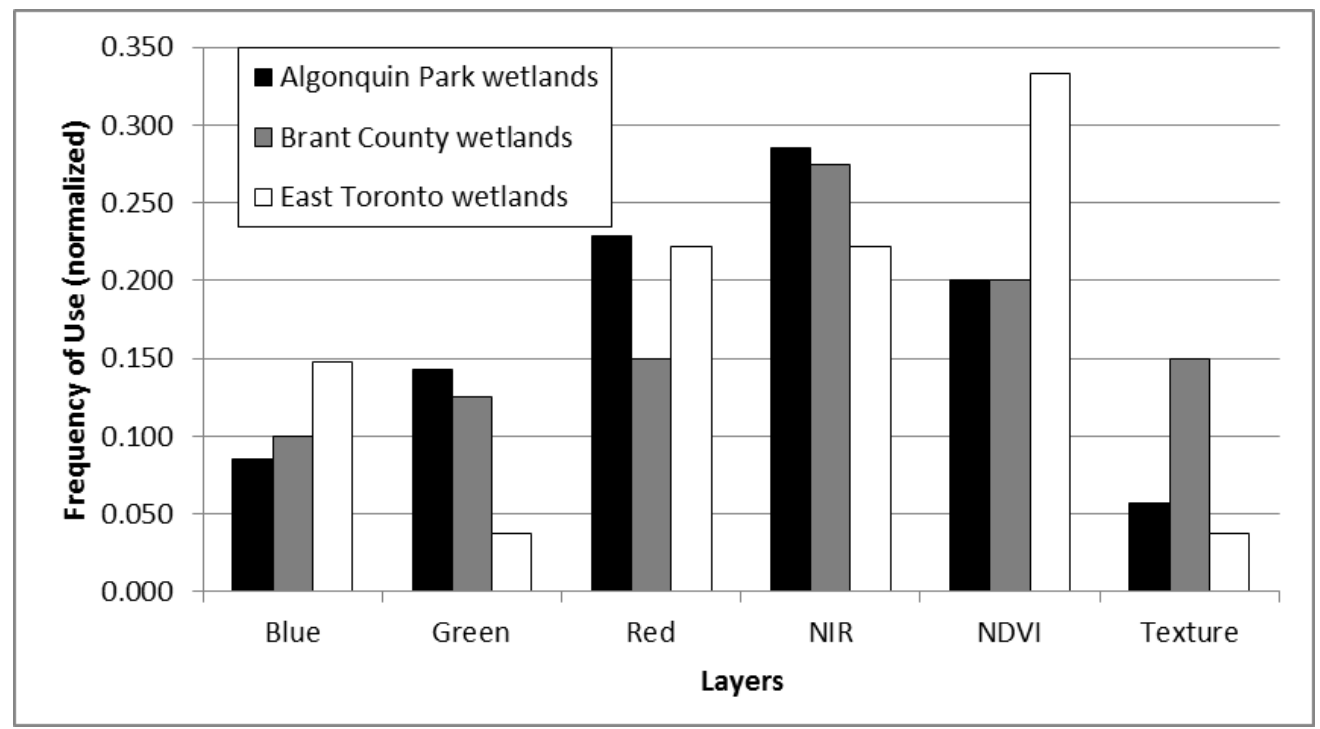

508 Figure 10. Comparison of mean object layer values providing the best separation between wetland 509 classes and all other landcover classes at each study site. Y-axis is showing the number of times a 510 layer provided the best separation distance between classes, normalized out of 1 . Average values 511 are standardized across total number of landcover classes at each site. 

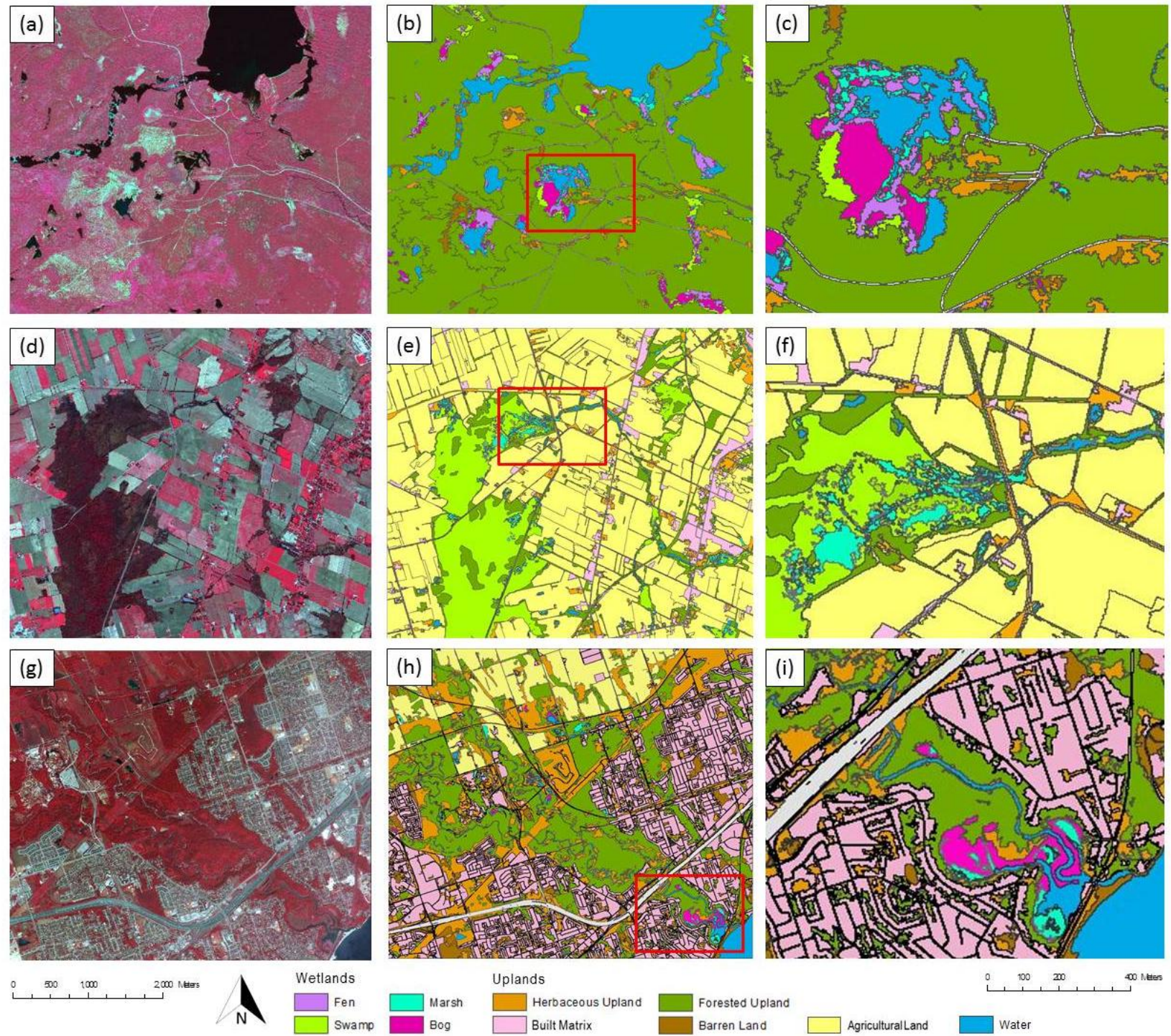

Uplands
$\begin{aligned} & \square \text { Herbaceous Upland } \\ & \square \text { Built Matrix }\end{aligned}$

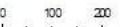

513 Figure 11. Classification results showing original satellite image (RBG: 432), final classified map, 514 and subset of classified map over the Algonquin Park natural site (a, b, c), the Brant county rural-

515 agricultural site (d, e, f), and the east Toronto urban site (g, h, i).

\section{Discussion}

517 In this study we examined the accuracy of a multi-scale GEOBIA approach in correctly classifying

518 wetlands across three different landscapes. Despite the variability in study areas, overall wetland

519 class accuracy across scenes was greater than $80 \%$ indicating that this methodological approach is

520 robust across scenes of varying heterogeneity due to human-disturbance. 


\section{Segmentation and the GEOBIA approach}

522 The multi-scale object-based approach provided an effective method of partitioning wetlands, and

523 other landcover classes. The class accuracy of wetlands (marsh, swamp, bog, fen) was higher than

524 grouped upland-wetland accuracy across all sites, which we attribute in part, to the use of the

525 hierarchical parent-child segmentation approach. The segmentation of whole wetland objects into

526 smaller objects for within wetland classification allowed this process to be constrained to its parent

527 class which minimizes the potential for misclassification with other groups. Repeatedly modifying

528 training objects to achieve the best classification also contributed to improving final map results.

529 Specifically, a sample of incorrectly classified objects should be iteratively selected as training

530 samples to retrain the classifier so that subsequent classifications can target areas of demonstrated

531 spectral overlap or confusion. The visual approach to selecting the scale parameter proved to be a

532 robust method drawing upon the inherent ability of the human eye to distinguish between

533 landscape elements and neighbourhood context. The use of the modified ED3 algorithm to evaluate

534 the scale parameter provided important quantitative support for scale selection, as well as further

535 information on the range of appropriate scale values. Thus, the combination of both quantitative

536 and qualitative measures are recommended as each is important for selecting the scale parameter.

537 At the coarse segmentation level, the addition of the NDVI layers resulted in a general

538 improvement in delineating boundaries of classes which were comprised of, or adjacent to

539 vegetation such as crop fields bordered by hedgerows, or mixed herbaceous vegetation.

540 Multispectral indices have been shown to improve models of wetland discrimination (Bradley \&

541 Fleishman, 2008) due to their sensitivity to vegetation surface roughness and phenological stage

542 (Davranche et al., 2010). Elevation information improved segmentation of whole wetland

543 boundaries at the medium scale, and particularly in palustrine (inland) wetlands as opposed to

544 lacustrine (lake-associated) wetlands. This is likely a result of a greater difference in elevation

545 between inland wetlands and the terrestrial uplands which completely surround them. The NDVI

546 layer contributed more to the segmentation of lacustrine wetlands, which were present in small

547 proportions in the park and urban scenes.

548 Texture contributed most to segmentation at the finest scale where the spatial information

549 improved delineation of wetland vegetation communities. Resultant image objects were larger than

550 those segmented without textural information, and also resulted in objects that more accurately

551 captured edges where macrophyte communities transitioned. Previous work has shown that texture

552 analysis can improve classification accuracy by reducing the confusion between permanent crops 
and perennial meadows (Peña-Barragán et al., 2011). For future work, we recommend the

554 exploration of higher order texture measures such as those derived from the grey-level co-

555 occurrence matrix (Haralick et al.,1973), which has shown success in discriminating between

556 deciduous and evergreen tree species (Kim et al, 2009) and may improve classification accuracy

557 between treed uplands and swamps (treed wetlands).

558 It should also be noted that the identified scale at each level cannot be interpreted as a universal

559 value that can be applied to any image of similar composition or resolution (spectral, spatial, and

560 radiometric). The size and shape of image objects is greatly affected by the extent, composition,

561 spectral heterogeneity and type of segmentation algorithm used. For example in preliminary

562 segmentation tests, it was found that a subset of a larger image segmented at a scale value of 100 ,

563 would create very different image objects than those created by segmenting the entire image at the

564 same scale of 100. Here, the extent alone alters the resultant objects relative to the scale value

565 which remains constant. Nevertheless, the scale values reported here are for the purpose of

566 comparison of targeted land cover classes within the study sites, and not as a recommendation for

567 optimal scale values to use for other images composed of similar elements as ours.

\section{Classification accuracy}

569 Wetlands in landscapes of varying heterogeneity were classified with an accuracy between $81 \%$

570 (kappa 0.78) and 90\% (kappa 0.88) with the least disturbed site achieving the highest accuracy.

571 While what is considered acceptable for mapping accuracy may vary, the recommended target of

$57285 \%$ overall accuracy (Foody, 2002; Thomlinson et al., 1999) was achieved by two of the three

573 classification maps. Not unexpectedly, differences in upland complexity resulted in varied

574 outcomes with regard to both segmentation and classification accuracy. When comparing overall

575 classification accuracy, there is a small but consistently poorer performance in the agricultural site

576 across all accuracy measures and grouping of classes, including wetlands.

577 A dominant contributor to mapping error was the confusion between the forested upland and

578 swamp class. The high proportion of swamp areas present in the agricultural landscape likely

579 contributed to the lower classification error. Here, the lower accuracy results were partly attributed

580 to the presence of facultative tree species such as Red maple (Acer rubrum) which can grow in

581 both saturated wetland soils and dry upland soils and would show spectral and textural similarity if

582 above ground reflectance does not reveal the hydrologic state beneath (Sader et al, 1995).

583 Confusion between agricultural land and herbaceous upland further reduced accuracy. Notably, all 
confused classes belonged to groups containing an abundance of vegetation, indicating the need for better measures to separate these similar classes. Similarly, the urban land cover map demonstrated reduced accuracy with the swamp class, despite (or partly as a result of) the low proportion of swamps in this scene. The use of advanced texture measures such as the grey level co-occurrence matrix, multi-date MS imagery or data from active sensors would likely help to improve accuracy over this class and should be investigated further.

Overall, it is generally accepted that mapping error on less frequent classes like wetlands will be higher than error on dominant classes (Cunningham, 2006). The relative rarity of wetlands at each site likely contributed to the over/under classification and to spectral confusion of wetlands classes. Wright \& Gallant (2007) documented a similar error for palustrine wetland mapping in Yellowstone National Park for which wetlands comprised less than $6 \%$ of the total cover.

The use of temporal imagery has been shown to improve wetland detection, and utilises a major advantage of Earth-observing satellite data. Dechka et al. (2002) classified prairie wetland habitats in southern Saskatchewan using two IKONOS images acquired 24 May 2000 and 29 July 2000 to maximize seasonal variation in vegetation growth from minimal spring conditions to mid-summer growth. The multi-temporal combination of May and July imagery produced the highest accuracy (95.9\%), although compared to results using the only the July image (84.4\%) authors concluded that the increase in accuracy may not be enough to justify the high cost of additional multitemporal image acquisitions. Interestingly, in this study the earlier season (May) image produced the lower classification accuracy (50.5\%), while others found that spring imagery was most optimal for wetland discrimination (Ozesmi \& Bauer, 2002; Gilmer et al., 1980). Dingle-Robertson (2014) examine Ontario wetland classification according to the Ontario Wetland Evaluation System across three seasons using WorldView2, Landsat5, and Radarsat2 data and found that high spatial resolution WorldView2 data from spring or summer acquisitions produced the highest accuracies. Other multi-temporal work has found improvement in the identification of wetland plant species using a combination of field spectral data, LiDAR top of canopy data, and multi-date Quickbird imagery (Gilmore et al., 2002), as well as improved accuracy in mapping seasonally flooded forested wetlands using multi-date RADARSAT data (Townsend, 2001). In this study, multi-date imagery was only available for two of the three sites (the urban east Toronto site only had high spatial resolution data available in July) therefore a multi-date evaluation was not possible. However, future work requiring higher classification accuracy, for example in wetland change detection studies, should consider employing a multi-temporal approach. 
Other factors may have influenced final classification accuracy such as the difference in timing of image acquisitions across study areas, and the difference in sensors. The Brant County agricultural site and the Algonquin park site were both acquired in the spring from the GeoEye 1 sensor (25 May 2013, and 9 April 2012 respectively), albeit in different years, whereas the east Toronto urban site was acquired in the summer (25 July 2012) from the WorldView2 sensor. From an operational standpoint, acquiring satellite imagery that perfectly matches the required timing and conditions mandated by the study, can present one of the greatest challenges in remote sensing research. Shifting priorities in commercial tasking orders, limited availability of archived imagery, presence of cloud cover, and high cost, can collectively contribute to mismatches in sensor and temporal continuity. Thus results from the urban scene may not be directly comparable to results from the agricultural and park scenes, as the presence of vegetation further along in development and growth, as well as the slightly narrower bandwidth of the red channel (630-390 nm WorldView2 compared to 655-690 nm GeoEye1), and the near infrared channel (770-895 nm WorldView2 compared to 780-920 nm GeoEye1) may have influenced final results. Yet, these differences can also have a positive effect if identifying potential benefits or disadvantages of one sensor configuration in comparison with the other. For example, it is interesting that for the landscapes represented by GeoEye1 imagery (park and agricultural), which operates with a wider NIR bandwidth, the contribution of this band to classification is greater than the NDVI layer. Conversely, for the urban site based on the narrower NIR band of WorldView2, the opposite is true. This raises the possibility that different sensors may utilise spectral layers differently in the classification process as a result of their bandwidth, and is a topic warrants further investigation. However, despite this discontinuity in sensor and image acquisition timing, accuracy results over the urban site were neither higher nor lower than the accuracy over the other two sites with matching sensors and dates. While this uncertainty should be recognized, I do not believe it negates the results provided in this study, and our results further demonstrate that wetland detection can be successfully achieved across landscapes of varying heterogeneity without the use of extensive ancillary data.

\section{Landscape heterogeneity}

A primary objective of this study was to examine if this methodological approach was robust to variability in scene heterogeneity caused by human-disturbance. Overall, with wetland accuracy results above $80 \%$ across all scenes, we concluded that this method was indeed well-suited to classifying wetlands from landscapes of varied heterogeneity, but there was a slight pattern of 
decreased accuracy with increasing scene complexity. Fahrig et al. (2011) made an important

649 distinction between compositional heterogeneity (a landscape with a greater variation of land cover

650 types) and configurational heterogeneity (a more complex spatial patterning of land cover types)

651 with which to describe a landscape. Although, it should be noted that there is no universally

652 accepted description of ecological heterogeneity (Cadenasso et al. 2007) which makes it difficult to

653 identify those regions for which special considerations should be taken.

654 In terms of land cover heterogeneity, Smith et al. (2002) quantified both landcover patch size and

655 heterogeneity over a large portion of the eastern US and demonstrated an almost continuous

656 decrease in accuracy as heterogeneity increased, suggesting that landscape characteristics should

657 be afforded the same consideration in accuracy assessments as those conducted on landcover

658 classes. We noticed a similar relationship between landcover heterogeneity and map accuracy

659 among our study sites with the natural site achieving the best results, and more disturbed sites

660 performing worse. The decreased accuracy over the agricultural site was not anticipated since built

661 features were considered more complex than agricultural fields, and coverage of built areas was

662 considerably higher in the urban region. Yet, the relative ease of segmentation and classification of

663 residential parcels in both disturbed landscapes indicated that this class may not contribute as much

664 to classification confusion as originally thought. Upon further examination of results, urban-built

665 features have greater spectral and textural distinction which separate them more easily from

666 spectrally and texturally similar vegetated land cover classes. A careful review of misclassified

667 objects indicated that the wide range of upland vegetation classes of both human and natural origin,

668 may be responsible for the lower map accuracy in the rural site. This was especially true along

669 transitional areas where one dominant class transitioned into another. Cingolani et al. (2004)

670 experienced a similar challenge in mapping heterogeneous rangeland ecosystems where the

671 influence of grazed lands combined with natural environmental gradients to create complex

672 vegetated patterns that were difficult to separate.

\section{Conclusions}

674 A simple yet efficient methodology procedure for mapping wetlands across landscapes of varied 675 heterogeneity was presented in this paper. High spatial resolution satellite data and the GEOBIA 676 approach can be combined to provide a sound methodology for characterizing whole wetlands and 677 individual wetland classes. The GEOBIA approach specifically, was very appropriate for wetland 678 detection as it allowed for a nested multi-scale approach to constrain classification of wetland 
679

680

681

682

683

684

685

686

687

688

689

690

691

692

693

694

695

696

697

698

699

700

701

702

703

704

705

706

707

708 components to within defined wetland boundaries. In regards to landscape variations, a more heterogeneous landscape may negatively affect accurate wetland classification due to increased spatial and compositional complexity. Specifically, rural landscapes presented special challenges due to the large proportion of vegetated upland classes of both anthropogenic and natural origin that reduced segmentation accuracy and resulted in greater spectral overlap during the classification.

Future work in wetland mapping of treed swamp wetlands should include SAR data which can capture the presence of standing water underneath tree canopies. In all cases, image acquisition during early spring leaf-off conditions are recommended to aid in discriminating between confounding vegetation classes, though from an operational standpoint reliance on archived imagery often means that this is not possible.

Overall, the trend of reduced wetland coverage with increasing landscape complexity due to human disturbance creates ongoing challenges for accurate wetland delineation. Wetlands are important ecosystems contributing an estimated $40 \%$ of the value of global ecosystem services (Zedler, 2003) and a better recognition of their value should be demonstrated through stricter legislation for wetland protection, particularly where new developments are concerned. In many areas a previous wetland inventory may not exist, making identification, evaluation and monitoring of wetlands a challenge. This study demonstrated a robust approach to delineating wetlands across variable landscapes which can provide starting information for better management of these ecosystems. The multi-temporal aspect of satellite sensors can be exploited to provide repeat coverage allowing for change detection and evaluation of wetland health over time. However, the greater issue at hand is the ongoing loss and degradation of wetlands worldwide, which will have serious consequences for global climate as well as the maintenance of biodiversity.

\section{References}

Anderson, J.E., and Perry, J.E. (1996). Characterization of wetland plant stress using leaf spectral reflectance: Implications for wetland remote sensing. Wetlands, 16, 477-487.

Anderson, J. R., Hardy, E. E., Roach, J. T., \& Witmer, R. E. (1976). A Land Use And Land Cover Classification System For Use With Remote Sensor Data. A Revision of the Land Use Classification System as Presented in U.S. Geological Survey Circular 671, 964, 41. 
Benz, U. C., Hofmann, P., Willhauck, G., Lingenfelder, I., \& Heynen, M. (2004). Multi-resolution, object-oriented fuzzy analysis of remote sensing data for GIS-ready information. ISPRS Journal of Photogrammetry and Remote Sensing, 58, 239-258.

Berberoğlu, S., Akin, a., Atkinson, P. M., \& Curran, P. J. (2010). Utilizing image texture to detect land-cover change in Mediterranean coastal wetlands. International Journal of Remote Sensing, 31(11), 2793-2815.

Blaschke, T. (2010). Object based image analysis for remote sensing. ISPRS Journal of Photogrammetry and Remote Sensing, 65(1), 2-16.

Blaschke,T., G. J. Hay, M. Kelly, S. Lang, P. Hofmann, E. Addink, R. Q. Feitosa, F. van der Meer, H. van der Werff, F. van Coillie, and D. Tiede, 2014. Geographic Object-Based Image Analysis - Towards a new paradigm, ISPRS Journal of Photogrammetry and Remote Sensing, 87(1), 180-191.

Brock, M. A., Smith, R. G. B., \& Jarman, P. J. (1999). Drain it , dam it : alteration of water regime in shallow wetlands on the New England Tableland of New South Wales, Australia. Wetlands Ecology and Management, 7, 37-46.

Cadenasso, M. L., Pickett, S. T. a, \& Schwarz, K. (2007). Spatial heterogeneity in urban ecosystems: reconceptualizing land cover and a framework for classification. Frontiers in Ecology and the Environment, 5(2), 80-88.

Castillejo-González, I. L., López-Granados, F., García-Ferrer, A., Peña-Barragán, J. M., JuradoExpósito, M., de la Orden, M. S., \& González-Audicana, M. (2009). Object- and pixel-based analysis for mapping crops and their agro-environmental associated measures using QuickBird imagery. Computers and Electronics in Agriculture, 68, 207-215.

Cingolani, A. M., Renison, D., Zak, M. R., \& Cabido, M. R. (2004). Mapping vegetation in a heterogeneous mountain rangeland using landsat data: An alternative method to define and classify land-cover units. Remote Sensing of Environment, 92, 84-97.

http://doi.org/10.1016/j.rse.2004.05.008

Clinton, N., Holt, A., Scarborough, J., Yan, L., \& Gong, P. (2010). Accuracy Assessment Measures for Object-based Image Segmentation Goodness. Photogrammetric Engineering \& Remote Sensing, 76(3), 289-299.

Congalton, R. G. (1991). A review of assessing the accuracy of classifications of remotely sensed data. Remote Sensing of Environment, 37, 35-46.

Corcoran, J. M., Knight, J. F., \& Gallant, A. L. (2013). Influence of multi-source and multitemporal remotely sensed and ancillary data on the accuracy of random forest classification of wetlands in northern Minnesota. Remote Sensing, 5, 3212-3238.

Davranche, A., Lefebvre, G., \& Poulin, B. (2010). Wetland monitoring using classification trees and SPOT-5 seasonal time series. Remote Sensing of Environment, 114, 552-562. 
Dillabaugh, K. a, \& King, D. J. (2008). Riparian marshland composition and biomass mapping using Ikonos imagery. Canadian Journal of Remote Sensing, 34(2), 143-158.

Dingle Robertson, L., \& King, D. J. (2011). Comparison of pixel- and object-based classification in land cover change mapping. International Journal of Remote Sensing, 32(6), 1505-1529.

Dissanska, M., Bernier, M., \& Payette, S. (2009). Object-based classification of very high resolution panchromatic images for evaluating recent change in the structure of patterned peatlands. Canadian Journal of Remote Sensing, 35(2), 189-215.

Drăguţ, L., Csillik, O., Eisank, C., \& Tiede, D. (2014). Automated parameterisation for multi-scale image segmentation on multiple layers. ISPRS Journal of Photogrammetry and Remote Sensing, 88, 119-127.

Drăguţ, L., Tiede, D., \& Levick, S. R. (2010). ESP: a tool to estimate scale parameter for multiresolution image segmentation of remotely sensed data. International Journal of Geographical Information Science, 24(6), 859-871.

Duro, D. C., Franklin, S. E., \& Dubé, M. G. (2012). A comparison of pixel-based and object-based image analysis with selected machine learning algorithms for the classification of agricultural landscapes using SPOT-5 HRG imagery. Remote Sensing of Environment, 118, 259-272.

Fahrig, L., Baudry, J., Brotons, L., Burel, F. G., Crist, T. O., Fuller, R. J., ... Martin, J. L. (2011). Functional landscape heterogeneity and animal biodiversity in agricultural landscapes. Ecology Letters, 14, 101-112.

Foody, G. M. (2002). Status of land cover classification accuracy assessment. Remote Sensing of Environment, 80, 185-201.

Fournier, R. a, Grenier, M., Lavoie, A., \& Hélie, R. (2014). Towards a strategy to implement the Canadian Wetland Inventory using satellite remote sensing. Canadian Journal of Remote Sensing, 33(sup1), S1-S16.

Fournier, R. a., Grenier, M., Lavoie, A., \& Hélie, R. (2007). Towards a strategy to implement the Canadian Wetland Inventory using satellite remote sensing. Canadian Journal of Remote Sensing, 33, 1-16.

Gibbs, J. P. (1993). School of Forestry and Environmental Studies Yale University 205 Prospect St. New Haven, C T 06511. Wetlands, 13(1), 25-31.

Grenier, M., Demers, A. M., Labrecque, S., Benoit, M., Fournier, R. a., \& Drolet, B. (2007). An object-based method to map wetland using RADARSAT-1 and Landsat ETM images: test case on two sites in Quebec, Canada. Canadian Journal of Remote Sensing, 33(1), S28-S45.

Group, N. W. W. (1997). The Canadian Wetland Classification System. Wetlands Research Centre. 
Halabisky, M. (2011). Object-based classification of semi-arid wetlands. Journal of Applied Remote Sensing, 5(1), 053511-13.

Haralick, R. M., Shanmugam, K., \& Dinstein, I. (1973). Textural Features for Image Classification. IEEE Transactions on Systems, Man, and Cybernetics, 3(6), 610-621.

Harken, J., \& Sugumaran, R. (2005). Classification of Iowa wetlands using an airborne hyperspectral image: a comparison of the spectral angle mapper classifier and an objectoriented approach. Canadian Journal of Remote Sensing, 31(2), 167-174.

Hirano, A., Madden, M., \& Welch, R. (2003). Hyperspectral Image Data for Mapping Wetland Vegetation. Wetlands, 23(2), 436-448.

Hopkinson, C., Chasmer, L. E., Sass, G., Creed, I. F., Sitar, M., Kalbfleisch, W., \& Treitz, P. (2005). Vegetation class dependent errors in LIDAR ground elevation and canopy height estimates in a boreal wetland environment. Canadian Journal of Remote Sensing, 31(2), 191206.

$\mathrm{Hu}, \mathrm{X}$. and Q. Weng. 2011. Impervious surface area extraction from IKONOS imagery using an object-based fuzzy method, Geocarto International, 26(1), 3-20.

Im, J., Jensen, J. R., \& Tullis, J. A. (2008). Object-based change detection using correlation image analysis and image segmentation. International Journal of Remote Sensing, 29(2), 399-423.

Jensen, J. R., Rutchey, K., Koch, M. S., \& Narumalani, S. (1995). Inland wetland change detection in the Everglades Water Conservatin Area 2A using a time series of normalized remotely sensed data. Photogrammetric Engineering \& Remote Sensing, 61(2), 199-209.

Johnston, R., \& Barson, M. (1993). Remote sensing of Australian wetlands: An evaluation of Landsat TM data for inventory and classification. Australian Journal of Marine and Freshwater Research, 44, 235-52.

Kim, M., Madden, M., \& Warner, T. A. (2009). Forest Type Mapping using Object-specific Texture Measures fr om Multispectral Ikonos Imagery : Segmentation Quality and Image Classification Issues, 75(7), 819-829.

Klemas, V. (2011). Remote Sensing Techniques for Studying Coastal Ecosystems: An Overview. Journal of Coastal Research, 27, 2-17.

Liu, Y., Bian, L., Meng, Y., Wang, H., Zhang, S., Yang, Y., ... Wang, B. (2012). Discrepancy measures for selecting optimal combination of parameter values in object-based image analysis. ISPRS Journal of Photogrammetry and Remote Sensing, 68, 144-156.

Midwood, J. D., \& Chow-Fraser, P. (2010). Mapping Floating and Emergent Aquatic Vegetation in Coastal Wetlands of Eastern Georgian Bay, Lake Huron, Canada. Wetlands, 30, 1141-1152. 
Myint, S. W., Gober, P., Brazel, A., Grossman-Clarke, S., \& Weng, Q. (2011). Per-pixel vs. objectbased classification of urban land cover extraction using high spatial resolution imagery. Remote Sensing of Environment, 115(5), 1145-1161.

Ozesmi, S., \& Bauer, M. (2002). Satellite remote sensing of wetlands. Wetlands Ecology and Management, 10, 381-402.

Peña-Barragán, J. M., Ngugi, M. K., Plant, R. E., \& Six, J. (2011). Object-based crop identification using multiple vegetation indices, textural features and crop phenology. Remote Sensing of Environment, 115, 1301-1316.

Powers, R. P., Hay, G. J., \& Chen, G. (2011). How wetland type and area differ through scale: A GEOBIA case study in Alberta's Boreal Plains. Remote Sensing of Environment, 117, 135145.

Prigent, C., Matthews, E., Aires, F., \& Rossow, W. B. (2001). Remote sensing of global wetland dynamics with multiple satellite data sets. Geophysical Research Letters, 28(24), 4631.

Rokitnicki-Wojcik, D., Wei, A., \& Chow-Fraser, P. (2011). Transferability of object-based rule sets for mapping coastal high marsh habitat among different regions in Georgian Bay, Canada. Wetlands Ecology and Management, 19(3), 223-236.

Sawaya, K., Olmanson, L. ., Heinert, N. J., Brezonik, P. L., \& Bauer, M. E. (2003). Extending satellite remote sensing to local scales: land and water resource monitoring using highresolution imagery. Remote Sensing of Environment, 88, 144 - 156.

Schmidt, K., \& Skidmore, A. (2003). Spectral discrimination of vegetation types in a coastal wetland. Remote Sensing of Environment, 85(1), 92-108.

Semlitsch, R. D., \& Bodie, J. R. (1998). Are small, isolated wetlands expendable? Conservation Biology, 12(5), 1129-1133.

Shanmugam, P., Ahn, Y. H., \& Sanjeevi, S. (2006). A comparison of the classification of wetland characteristics by linear spectral mixture modelling and traditional hard classifiers on multispectral remotely sensed imagery in southern India. Ecological Modelling, 194, 379394.

Smith, J. H., Wickham, J. D., Stehman, S. V, \& Yang, L. (2002). Impacts of patch size and landcover heterogeneity on thematic image classification accuracy. Photogrammetric Engineering and Remote Sensing, 68(1), 65-70.

Thomlinson, J. R., Bolstad, P. V., \& Cohen, W. B. (1999). Coordinating methodologies for scaling landcover classifications from site-specific to global: Steps toward validating global map products. Remote Sensing of Environment, 70, 16-28.

Townsend, P. A., \& Walsh, S. J. (2001). Remote sensing of forested wetlands : application of multitemporal and multispectral satellite imagery to determine plant community composition and structure in southeastern USA. Plant Ecology, 157, 129-149. 
Wang, L., Sousa, W. P., \& Gong, P. (2004). Integration of object-based and pixel- based classification for mapping mangroves with IKONOS imagery. International Journal of Remote Sensing, 25(24), 5655-5669.

Wei, A., \& Chow-Fraser, P. (2007). Use of IKONOS Imagery to Map Coastal Wetlands of Georgian Bay. Fisheries, 32(4), 164-173.

Whiteside, T., \& Ahmad, W. (2005). A comparison of object-oriented and pixel-based classification methods for mapping land cover of northern australia. Proceedings of the SSC2005 Spatial Intelligence, Innovation and Praxis: The National Biennial Conference of the Spatial Sciences Institute, Melbourne., 1225-1231.

Wright, C., \& Gallant, A. (2007). Improved wetland remote sensing in Yellowstone National Park using classification trees to combine TM imagery and ancillary environmental data. Remote Sensing of Environment, 107, 582-605. h

Xu, B., \& Gong, P. (2007). Land-use/land-cover classification with multispectral and hyperspectral E0-1 data. Photogrammetric Engineering and Remote Sensing, 73(8), 955-965.

Yan, G., Mas, J. F., Maathuis, B. H. P., Xiangmin, Z., \& Van Dijk, P. M. (2006). Comparison of pixel-based and object-oriented image classification approaches - a case study in a coal fire area, Wuda, Inner Mongolia, China. International Journal of Remote Sensing, 27(18), 40394055 .

Yang, J., He, Y., \& Weng, Q. (2015). An automated method to parameterize segmentation scale by enhancing intrasegment homogeneity and intersegment heterogeneity. IEEE Geoscience and Remote Sensing Letters, 12(6), 1282-1286.

Yu, Q., Gong, P., Clinton, N., Biging, G., Kelly, M., \& Schirokauer, D. (2006). Object-based Detailed Vegetation Classification with Airborne High Spatial Resolution Remote Sensing Imagery. Photogrammetric Engineering \& Remote Sensing, 72(7), 799-811. 
Click here to download high resolution image
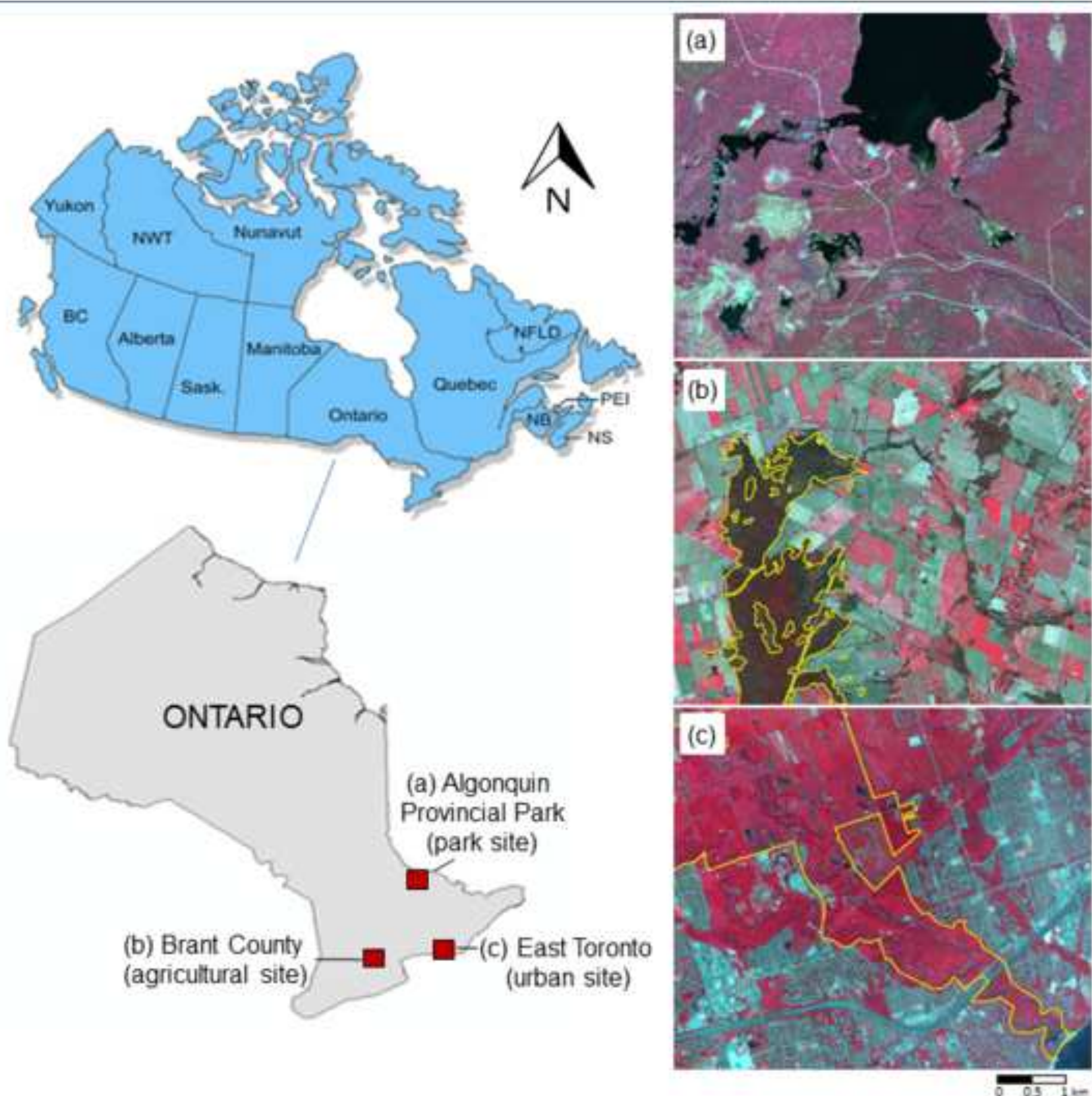


\section{Figure 2}

Click here to download high resolution image

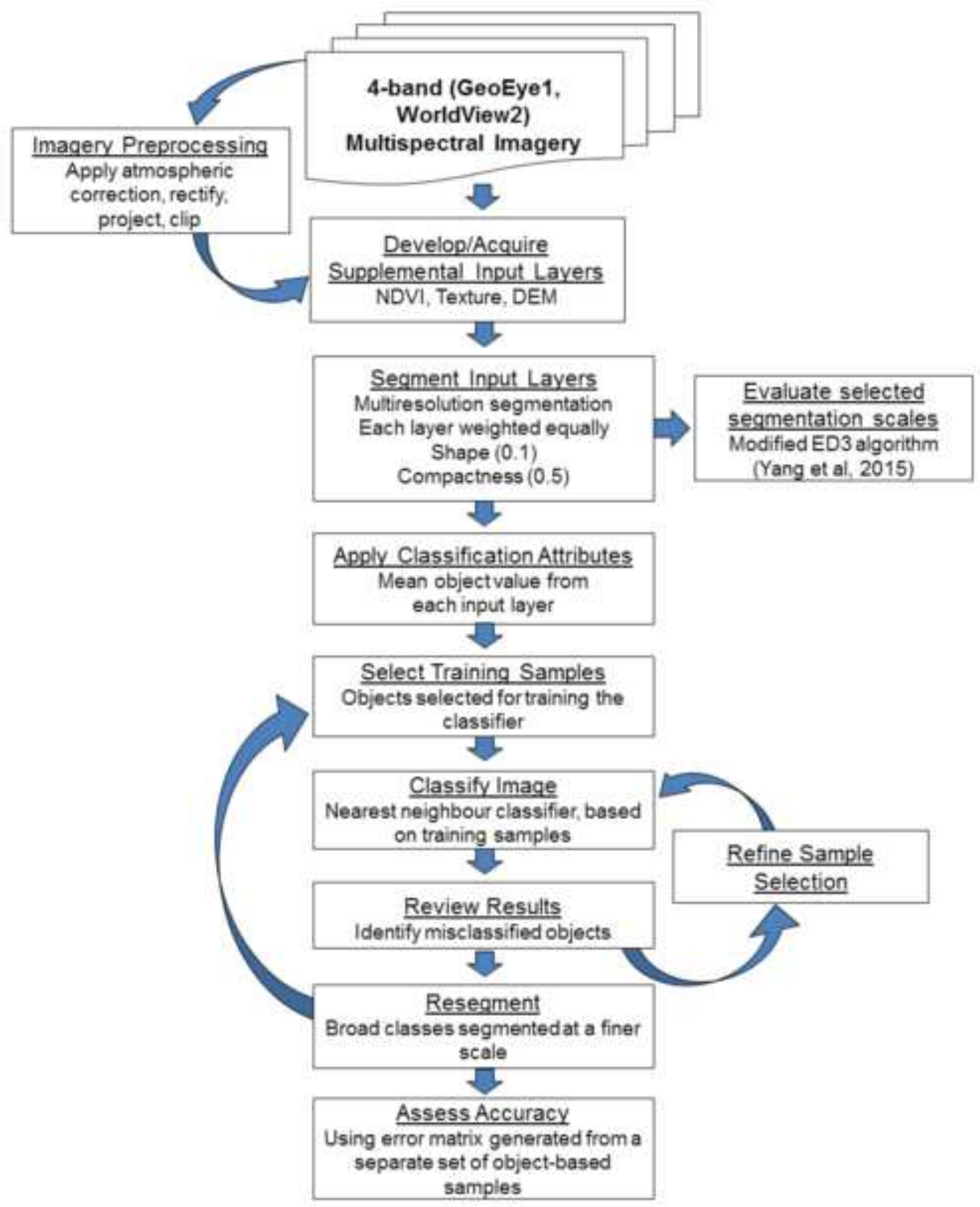


Click here to download high resolution image
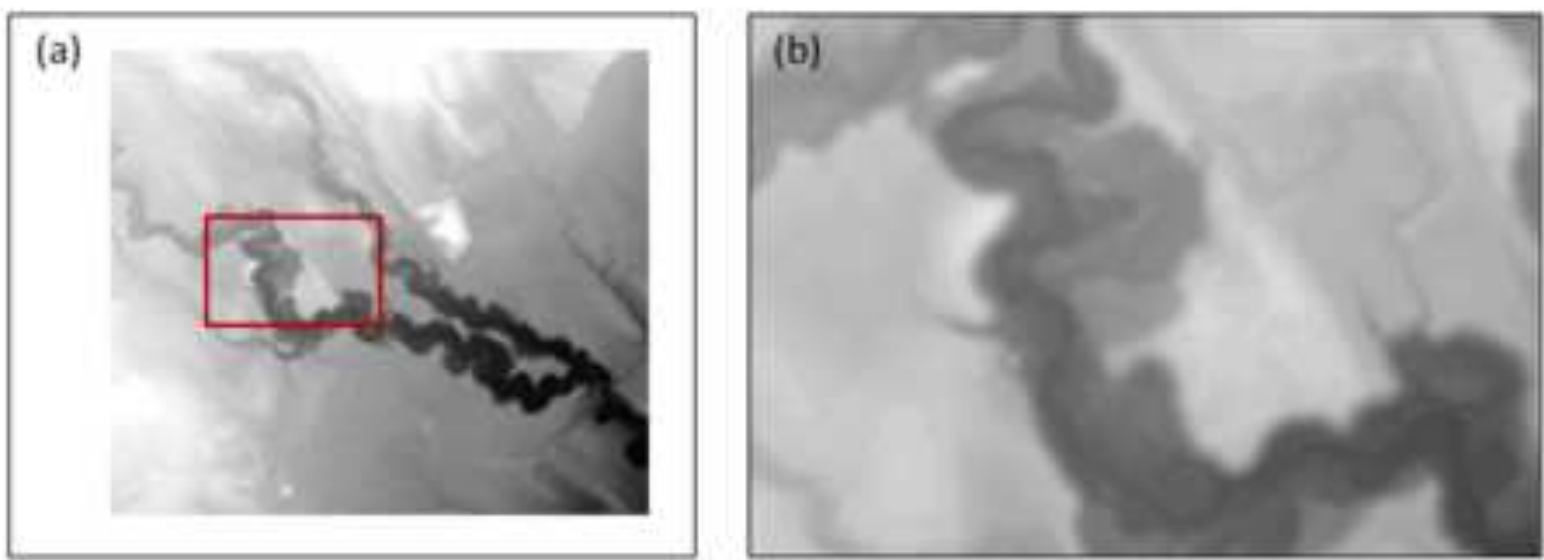

Elevation

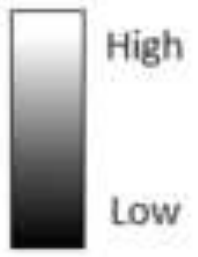

(c)

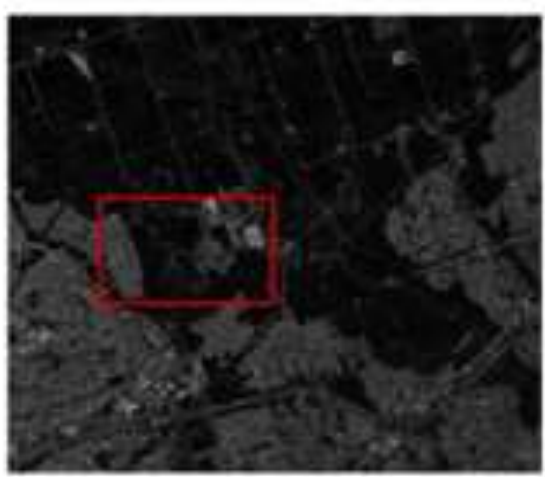

(e)
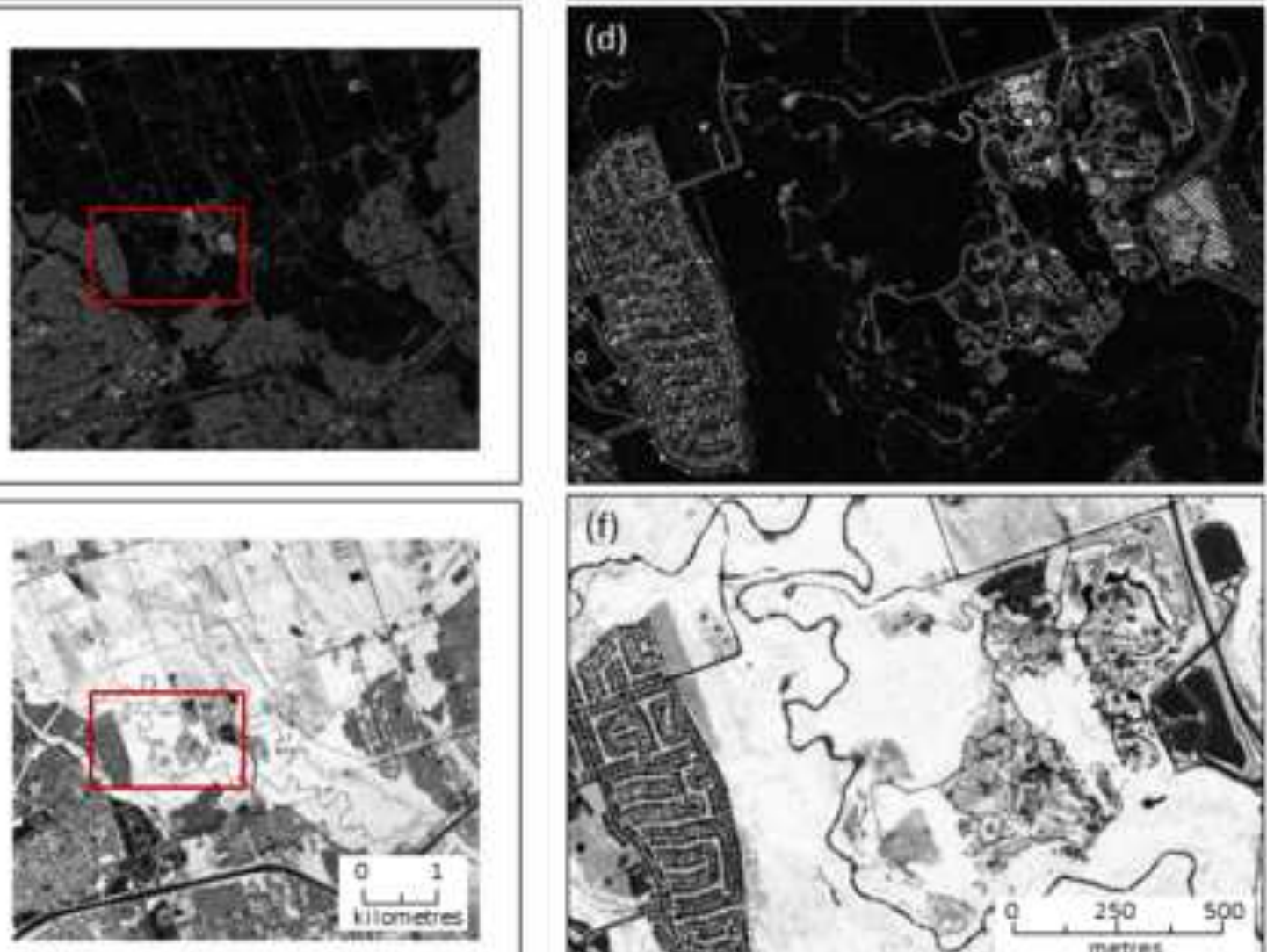

\section{Texture}
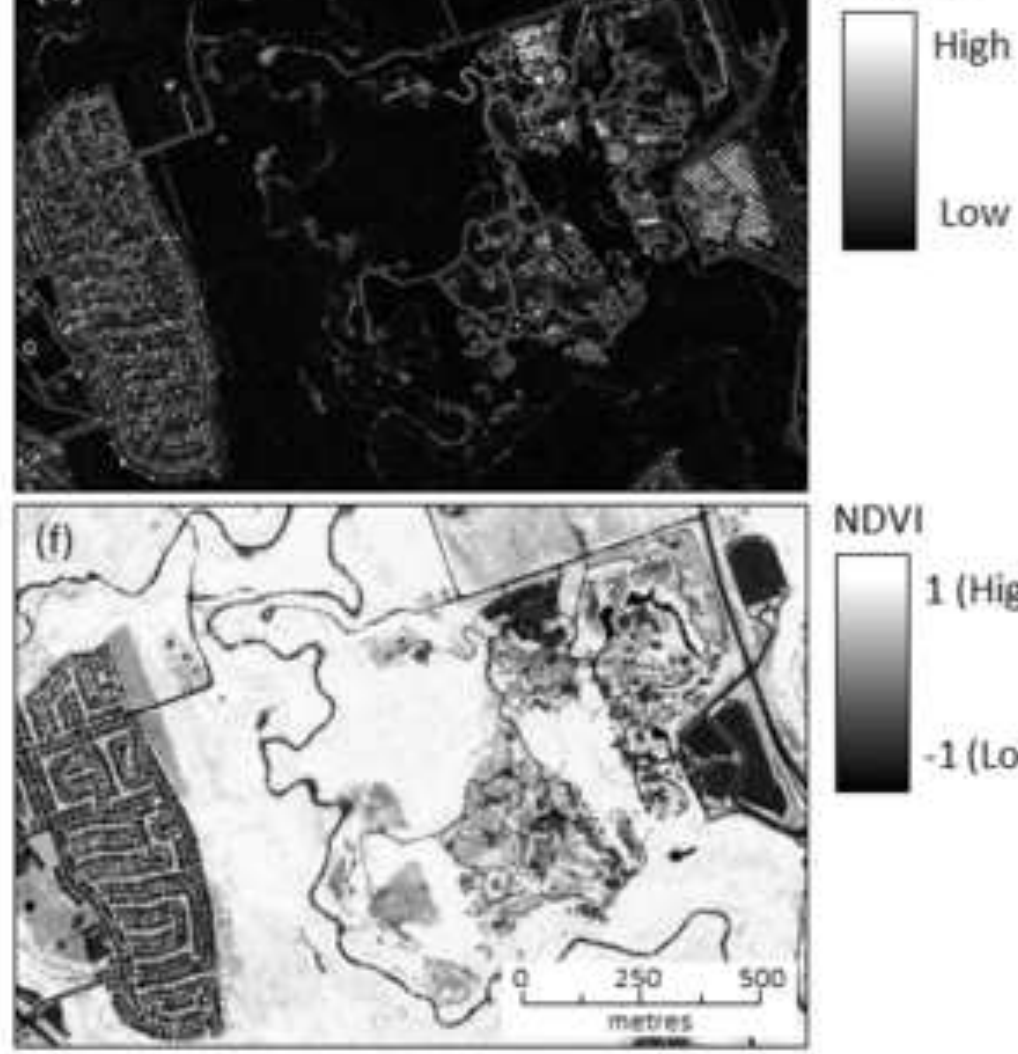

NDVI

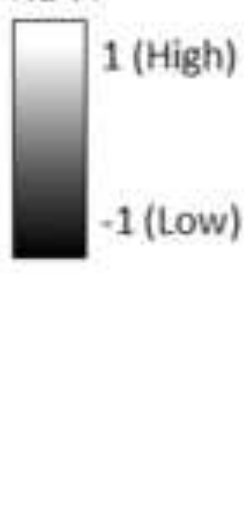




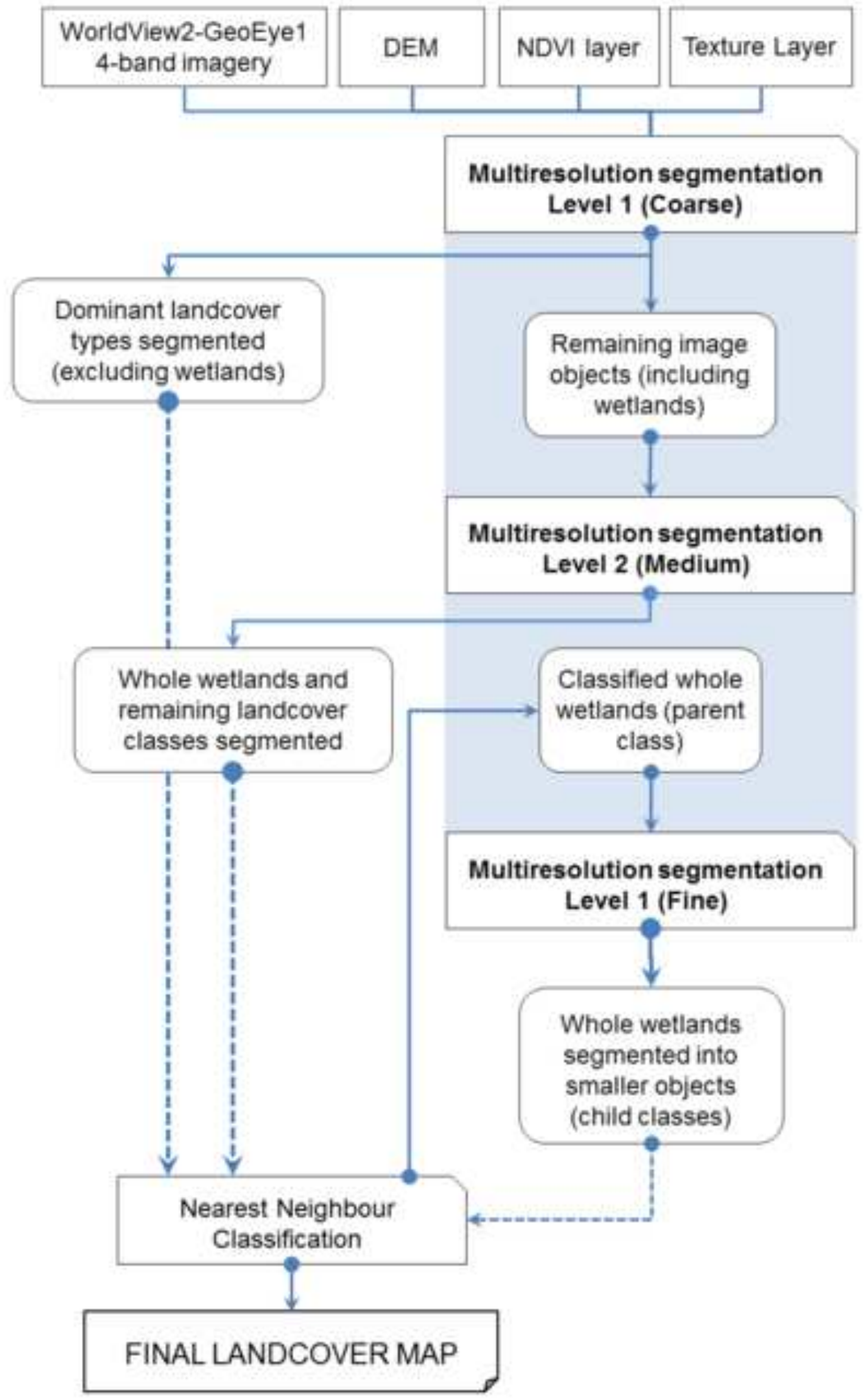




\section{Figure 5}

Click here to download high resolution image

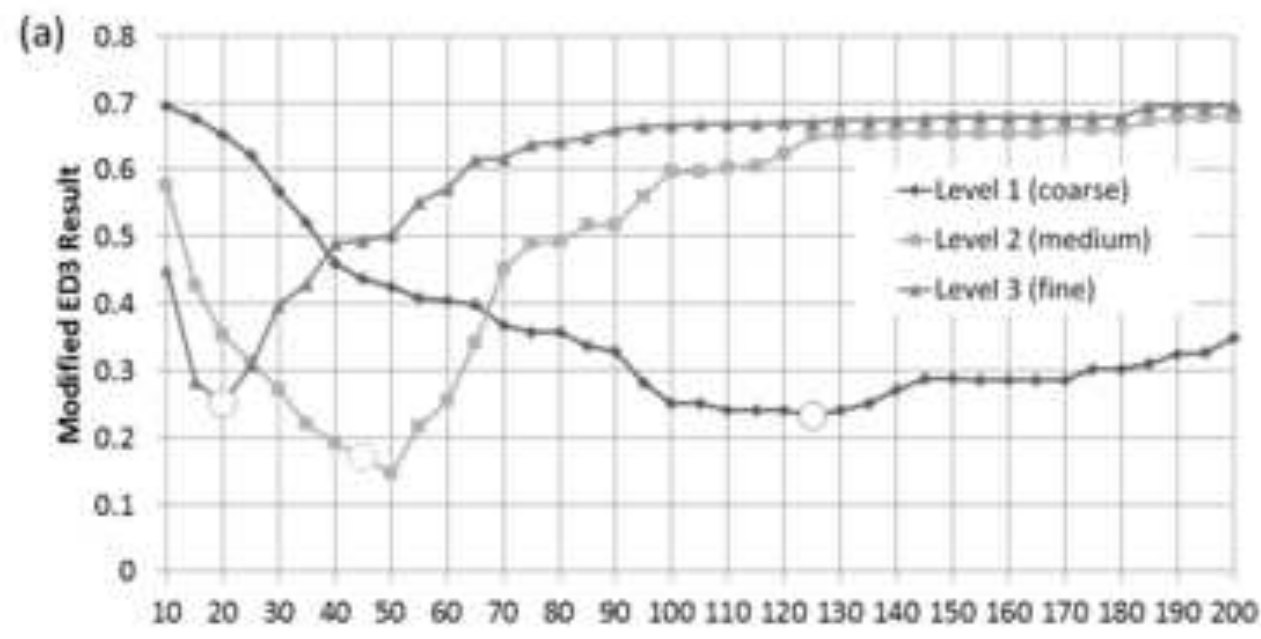

Multiresolution Segmentation Scale
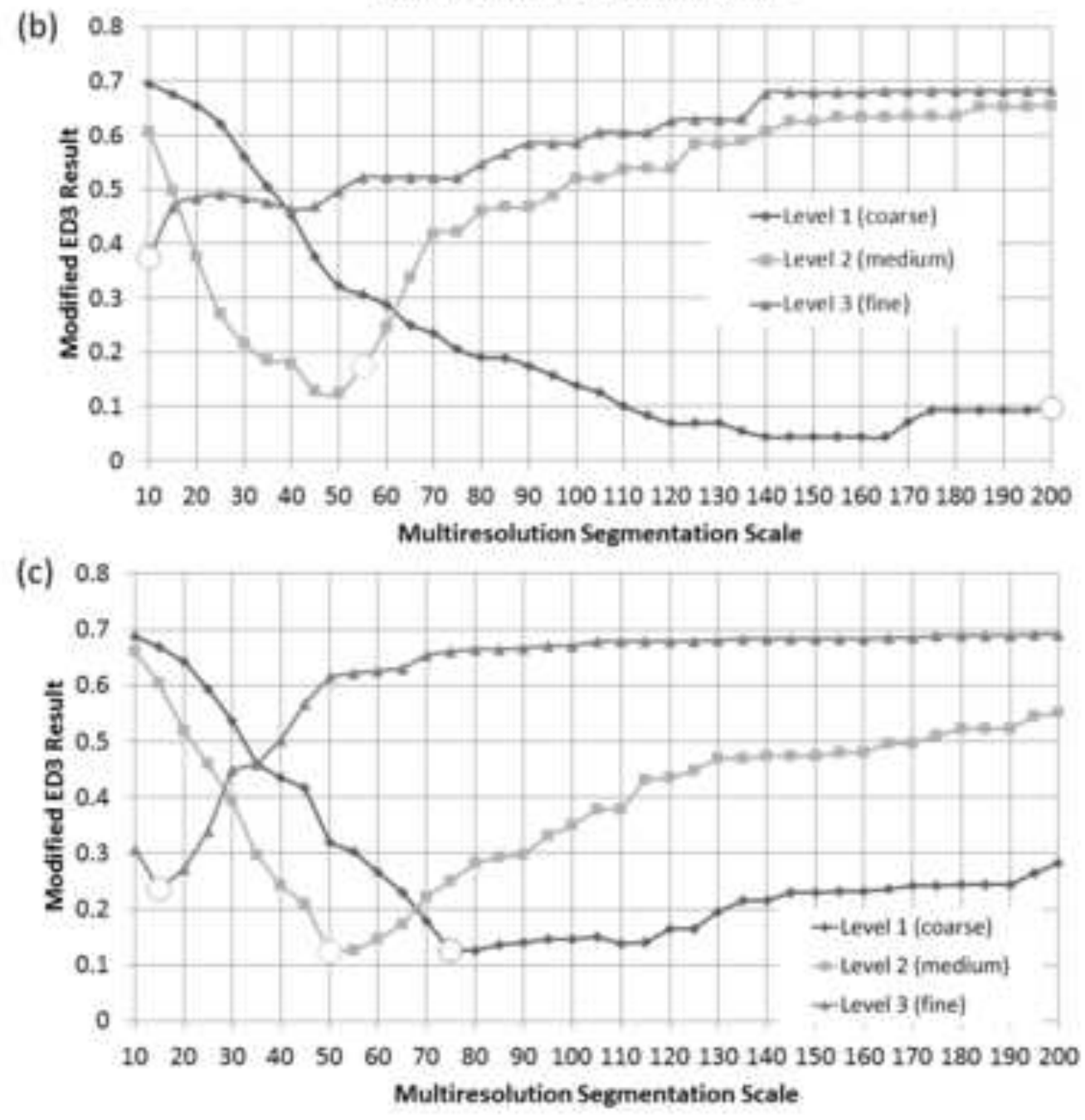

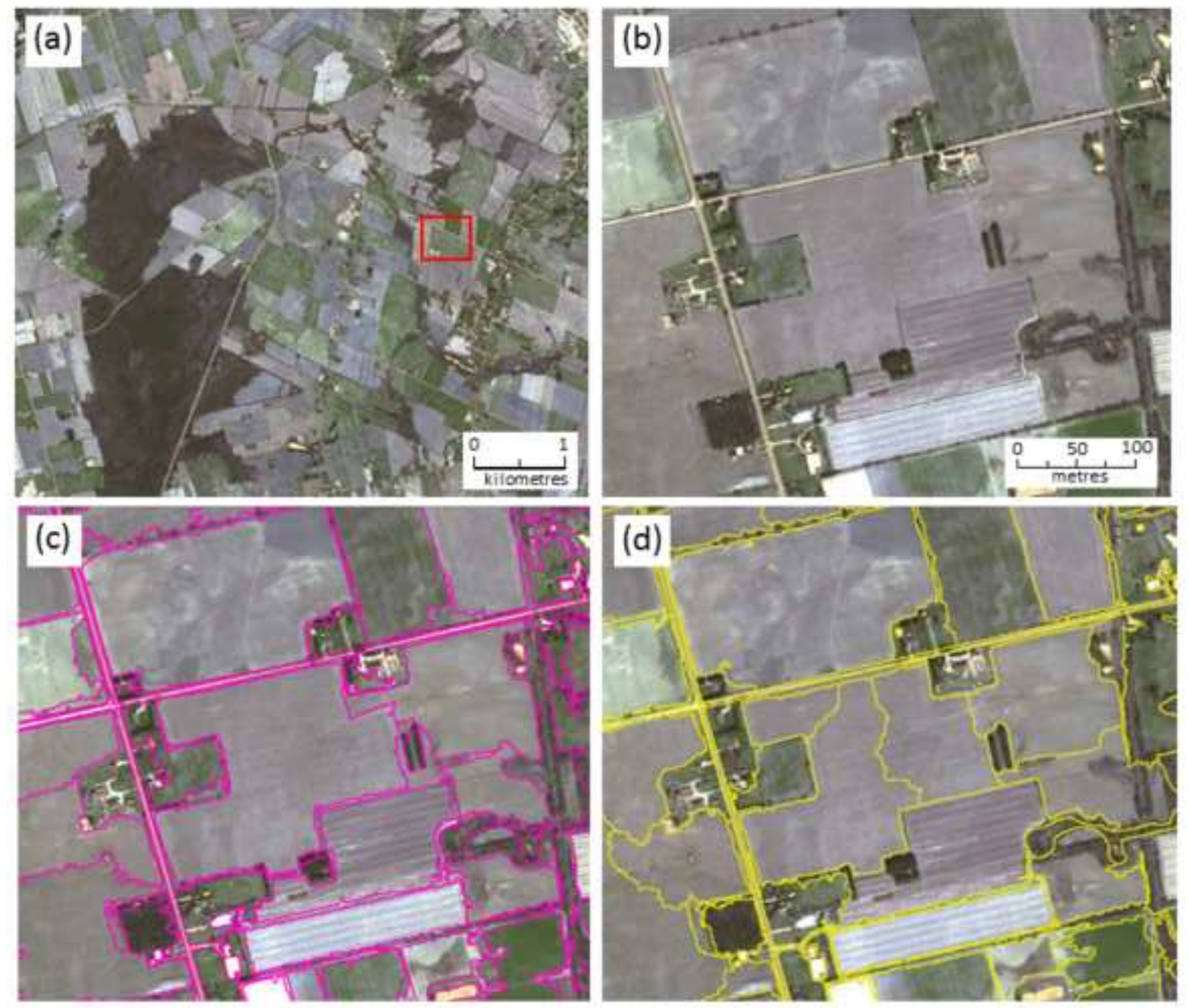

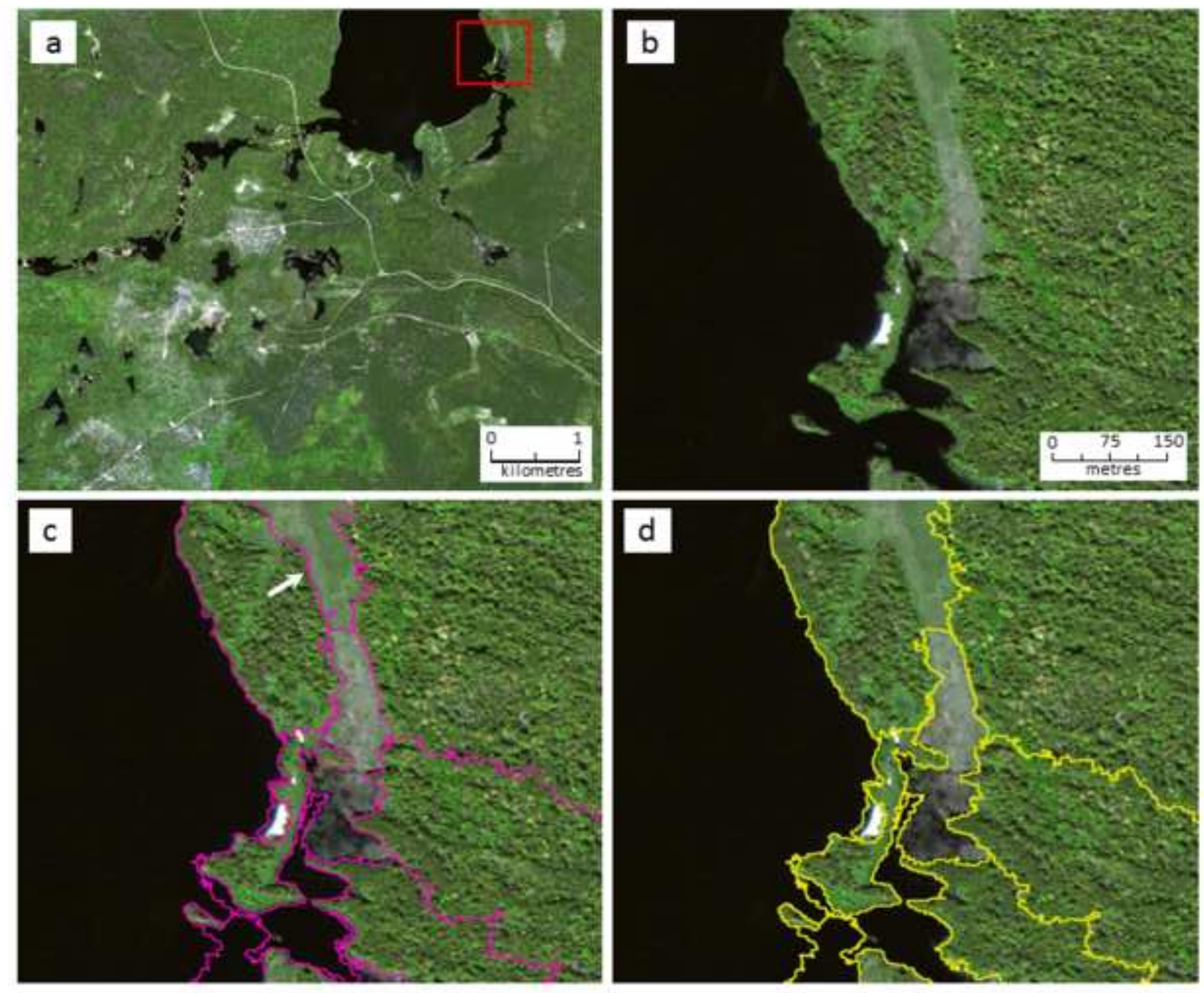
Figure 8
Click here to download high resolution image
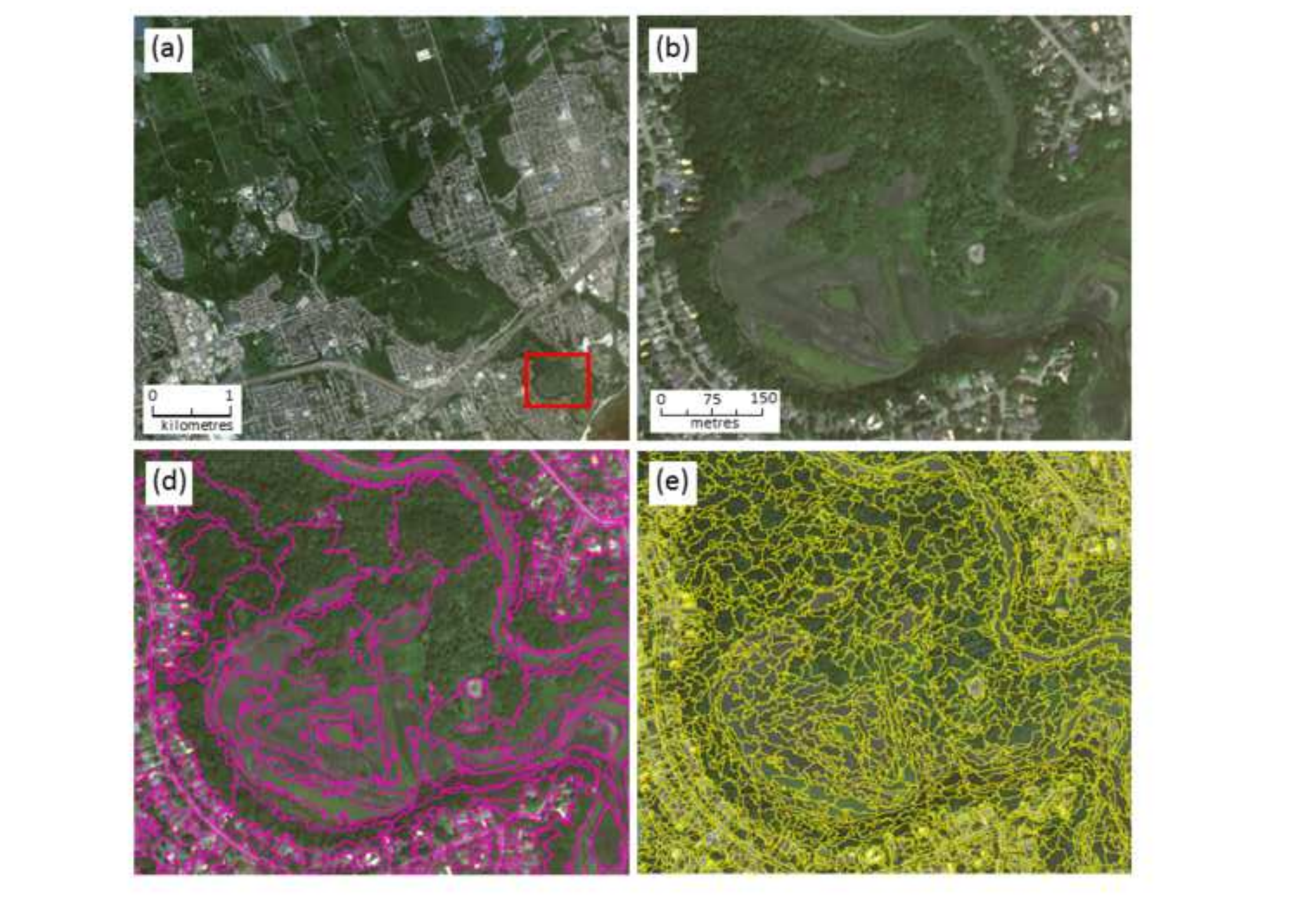
Figure 9

Click here to download high resolution image
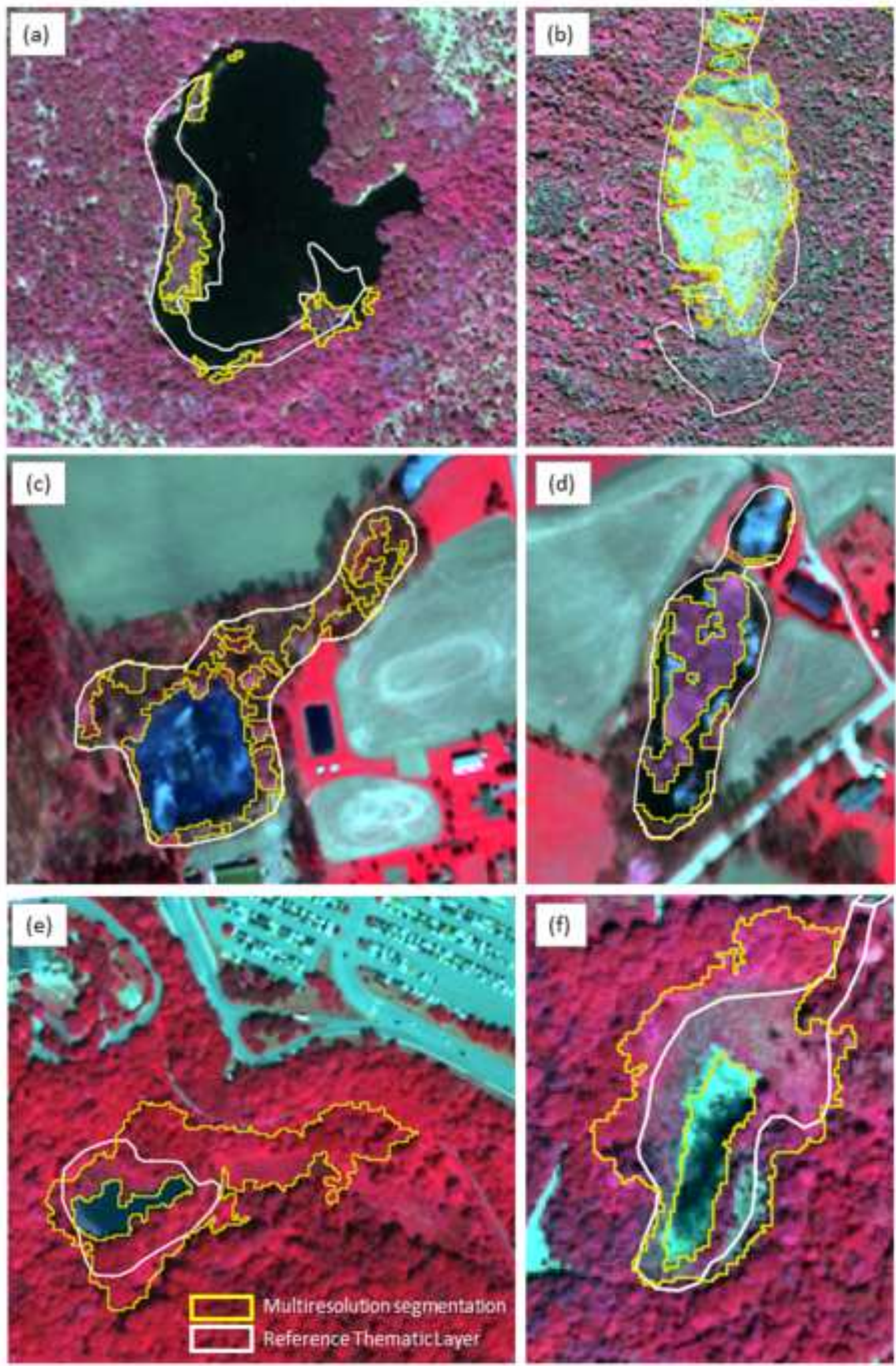
Click here to download high resolution image
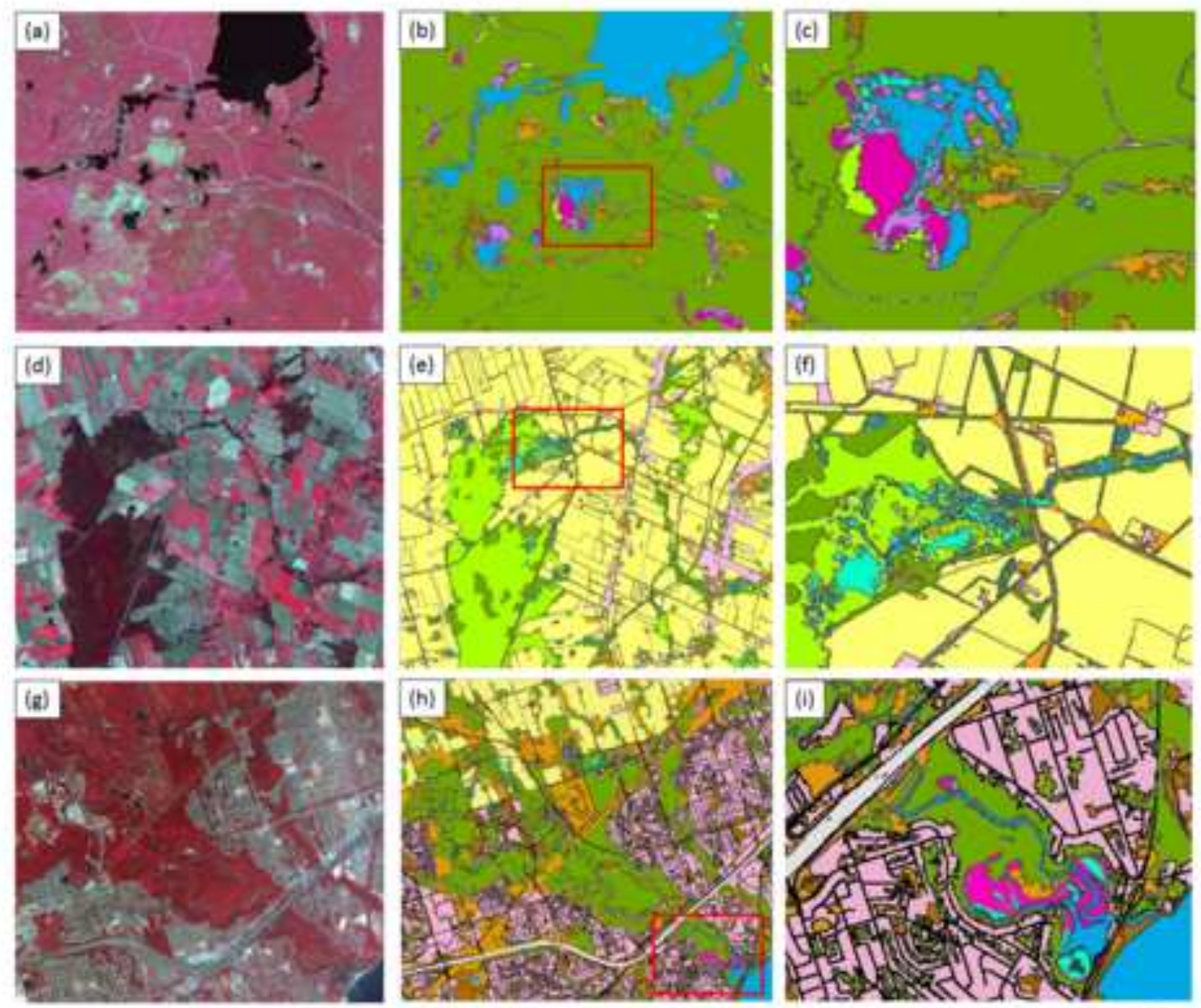

$=$

$-1$

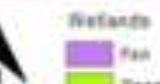

Mnimate

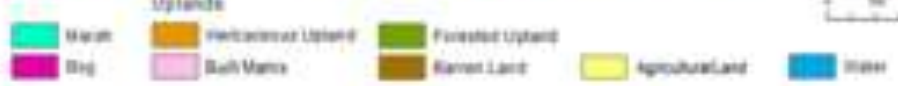

$+=$ a- 